\title{
Cauchy Families of Directional Distributions Closed Under Location and Scale Transformations
}

\author{
T.D. Downs*
}

Research Statistics, Inc., P.O. Box 840597, Houston, Texas 77284-0597, U.S.A.

\begin{abstract}
Directional statistics deals with angular data that come from non-linear objects such as circle circumferences or toroidal surfaces. A fundamental problem in directional statistics is that arithmetic cannot be meaningfully done on angles. Naive changes of location and scale like $\lambda^{\prime}=(\lambda-\mu) / \sigma$ for a spherical longitude $\lambda$ are inappropriate and often misleading since they are not interpretable as one-one mappings from a sphere onto itself. Finding ways to obtain angular scale changes and to construct families of spherical probability distributions that are closed under such scale changes have been unsuccessful. But, such families are successfully constructed herein by indirect but historically powerful methods. Thus, a unit sphere with a uniform probability distribution on its surface is centrally rotated to a suitable position, and then stereographically projected onto an extended complex plane, a linear surface especially amenable to directional and statistical computations. A central dilation is performed on the plane, the dilated plane is projected back in effect as a rescaled sphere, and the rescaled sphere is again rotated. This process induces a family of spherical Cauchy-type probability distributions on the sphere that is closed under composition of such processes (rotate sphere, project sphere to plane, dilate plane, project dilated plane back as a rescaled sphere, and rotate again). The distributions so induced can be generalized to higher dimensional spheres that are also closed under location and scale transformations. These distributions enjoy numerous interrelationships with one another and with linear and circular Cauchy distributions.
\end{abstract}

Keywords: Angular rescaling, Circular, Spherical and hyperspherical Cauchy distributions, Gauss' rotation image theorem, Möbius mapping, Polar, Mimetic, Extended and pseudo-polar decompositions, Spherical Möbius mapping, Spherical vonMises fisher distribution, Straight line and stereographic projections.

\section{INTRODUCTION}

Ordinary linear methods for statistically transforming and analyzing data are often useless and even misleading when applied to directional data. Alternative methods must be devised. The purpose of this paper is to investigate and develop methods for changing the scale or the amount of scatter in directional data. This should ideally be done with minimal distortion and loss of information in the data. These criteria seem to lead inexorably to Möbius functions as the vehicle for transforming the data, and these lead in turn to Cauchytype directional distributions.

Complex numbers are inherently directional by design and mathematically more tractable than the real numbers for dealing with directional data. Their analytical power far outweighs the relatively small investment required to get sufficiently familiar with a few complex techniques that are pertinent to directional statistics. These techniques are described and explained in section 2. Coordinate systems for spheres and planes are described in section 2.1. Transforming data back and forth between a complex plane and a sphere by stereographic projection is covered in section 2.2. Special Möbius mappings from the complex plane to itself or to another complex plane are described in section 2.3, and their spherical counterparts are de- scribed in section 2.4. Two

Address correspondence to the author at the Research Statistics, Inc., P.O. Box 840597, Houston, Texas 77284-0597, U.S.A.;

E-mail: proftddowns@msn.com special mappings are defined in section 2.5. Polar decompositions of $2 \times 2$ matrices whose entries are complex are defined in section 2.6 and 2.7. A summary of existing Cauchy distributional forms and the new circle form introduced herein are given in section 2.8.

Section 3 defines and constructs the probability distribution of the new "rescaled" circular Cauchy distribution, and compares it to the current "classical" circular Cauchy distribution. Section 4 defines and derives the new "rescaled" and "classical" spherical Cauchy distributions, as well as transformations and selected properties of these distributions. Section 5 provides discussions on selected related issues. Algorithms for the analysis and interpretation of spherical data appear in Appendices I and II. These pertain primarily to Möbius mappings, their associated decompositions and their relationships to the parameters of directional Cauchy distributions.

\section{METHODS AND MATERIALS}

\section{(1) Coordinate Systems}

Random spatial directions are taken to be points $z, z^{\mathrm{T}} z=$ 1 , on a unit $z$-sphere $S$ centered at the origin $0=(0,0,0)^{\mathrm{T}}$ of an $x y t$ rectangular coordinate system, and supplemented with spherical coordinates $z_{S}=(\theta, \varphi)=$ (colatitude, longitude), with $\theta \in[0, \pi]$ and $\varphi \in(-\pi, \pi]$. The linear $z$ and spherical $z_{\mathrm{S}}$ coordinates are related by

$z=(x, y, t)^{\mathrm{T}}=(\sin \theta \cos \varphi, \sin \theta \sin \varphi, \cos \theta)^{\mathrm{T}}$,

$z_{\mathrm{S}}=(\theta, \varphi)=(\operatorname{acos} t, \operatorname{atan} y / x)$,

where $\cos \varphi=x / r$, sin $\varphi=y / r$, and $r=\sin \theta=\left(x^{2}+y^{2}\right)^{1 / 2}$. 
Random planar directions are taken to be points $z_{\mathrm{C}}, z_{\mathrm{C}}=$ $\exp (\mathrm{i} \gamma)$, on unit circles of $S$ formed by the intersection of $S$ with complex planes passing through 0 . Mostly, though, spherical points $z$ which are constrained to great circles of $S$ are used to represent planar and spherical directions, facilitating shifting domains between circles and spheres.

Geographic terminology is used for $S$ : the north and south poles $n=(0,0,1)^{\mathrm{T}}$ and $s=-n$; the parallel circles of latitude; the great semi-circular meridians of longitude $\varphi$ joining $n$ and $s$, with origin the prime meridian; the great circles of longitude through $\pm \mathrm{s}$; latitude north or south of the equator; and colatitude $\theta, 0 \leq \theta \leq \pi$, with the central angle $\theta$ measured as deviation from $n$ or some specified point of $S$ such as a spherical mean direction $\mu, \mu^{\mathrm{T}} \mu=1$; the west hemisphere composed of $\varphi$-meridians with negative longitudes $\varphi$ $\in(-\pi, 0)$, and the east hemisphere with $\varphi$-meridians of nonnegative longitudes $\varphi \in(0, \pi)$. The two hemispheres are bordered by the great circle of longitude that is composed of the 0 -meridian (prime meridian) and the $\pi$-meridian.

The horizontal $x y$-plane, denoted by $E$, is considered as an extended complex plane with $\infty$ the sole improper point. Complex numbers $z_{\mathrm{E}}$ in any extended complex plane may be expressed as $z_{\mathrm{E}}=r \exp (\mathrm{i} \varphi)$ or $z_{\mathrm{E}}=x_{\mathrm{E}}+\mathrm{i} y_{\mathrm{E}}$, where $r^{2}=x_{\mathrm{E}}^{2}+$ $y_{\mathrm{E}}^{2}$ and $\mathrm{i}^{2}=-1$. The origin in $E$ is also denoted by 0 . The equator of $S$ coincides with the unit circle $U$ of $E$. All straight lines in any extended complex plane are extended lines and have in common the single improper point $\infty$. Horizontal directions from points on the polar axis of $S$ are identified and located by points $z=\exp (\mathrm{i} \varphi)$ on $U$, or by their longitudes $\varphi$.

Any great circle of longitude $C$ is located and oriented by the longitude $\varphi$ of its east meridian. Each such circle $\mathrm{C}$ has a unique vertical extended complex plane $F$ whose unit circle is $C$ and whose location and orientation are the same as those of $C$. Each vertical plane $F$ intersects $E$ in a center line $L$ (a straight line that passes through 0 in an extended complex plane). Each vertical plane $F$ contains the polar axis \pm s along the $t$-axis. The origins of $E, F$ and the $x y t$ coordinate space are identical to one another and also to the common centers of $U$, each unit circle $C$, and $S$.

Every unit circle $C$ in any distribution in this paper is taken, for theoretical purposes, to be a unit circle on $S$, but this does not preclude such distributions on stand-alone circles or circles in other settings. Points on the unit circle of any extended complex plane may be expressed globally as spherical points $z$ or $z_{\mathrm{S}}$ constrained to lie on $C$, or locally as complex circular points $z_{\mathrm{C}}=\exp (\mathrm{i} \gamma)$, or as angles, $\gamma, \gamma \in$ $(-\pi, \pi]$, with a convenient origin on $C$. In the latter case each point of $C$ is identified with its unique local angular designation $\gamma$. Similarly, local linear coordinates $x_{\mathrm{L}}$ for points on a center line $L$ are identified with the points on $L$, and we write:

$$
\begin{aligned}
& C=\{z ; z \in C \subset S\}=\left\{z_{\mathrm{C}} ; z_{\mathrm{C}}=\exp (\mathrm{i} \gamma)\right\}=\{\gamma ; \gamma \in(-\pi, \pi]\}, \\
& L=\left\{x_{\mathrm{L}} ; x_{\mathrm{L}} \in(-\infty, \infty]\right\},
\end{aligned}
$$

where $C$ and $L$ lie in extended complex planes. The location of a center line $L$ in the horizontal plane $E$ is fixed by the longitude $\varphi, \varphi \in[0, \pi)$, of its non-negative east ray with points $x_{\mathrm{L}}>0$ (and with $x_{\mathrm{L}}<0$ on the west ray).
A central rotation of $S$ is expressed as $A S$, where $A$ is a $3 \times 3$ orthogonal matrix with determinant $|A|= \pm 1$. The rotation is proper, or improper according as $|A|=+1$, or -1 . Objects on $S$ such as a point $z$ or a great circle $C$ may be considered as rotated along with $S$, and their new positions are denoted by $A z$ or $A C$. Other objects intersecting $S$ but not on $S$, such as the horizontal plane $E$, any vertical plane $F$, or coordinate axes may also be considered as being centrally rotated along with $S$ when convenient.

The spherical point $\mu, \mu^{\mathrm{T}} \mu=1$, represents the mean direction for a spherical probability distribution on $S$, and also represents the common mean direction for all the great circles on $S$ with circular probability distributions that pass through $\pm \mu$. There is at most one mean direction point $\mu$ on the sphere $S$ at any given time.

Different domains must simultaneously be dealt with from time to time. Instead of creating a hodgepodge of notation for each such situation or combination of situations, we will create adaptive notations applicable to most situations. In particular, the point symbols $\mu$ and $z$ will have different meanings according to the context in which they appear, and it will be clear from that context which of the following interpretations of $\mu$ and of $z$ are appropriate:

$\mu=\mu_{\mathrm{C}}=\exp (\mathrm{i} u)=u$ for a mean direction on a unit circle $C$, $=\left(\mu_{\mathrm{x}} \mu_{\mathrm{y}} \mu_{\mathrm{t}}\right)^{\mathrm{T}}$ for a mean direction point on $S$.

$$
\begin{aligned}
z & =z_{\mathrm{C}}=\exp (\mathrm{i} \gamma)=\gamma \text { for a point on a unit circle } C, \\
& =(x y t)^{\mathrm{T}} \text { for a point on } S .
\end{aligned}
$$

A useful notational convention makes it easier to assign coordinate values to points on unit spheres or circles.

If a mean direction $\mu$ exists on $S$, then, unless stated otherwise, $\pm \mu$ take precedence globally over $\pm n$ for spherical coordinates: colatitude $\theta$ is measured as the angular deviation from $\mu$, not from $n$; the great circles of longitude and the vertical extended complex planes containing them are considered as the great circles and extended complex planes that pass through $\pm \mu$, not $\pm n$; and the parallel circles of latitude including the equator are now orthogonal to the mean axis through $\pm \mu$, not to the polar axis through $\pm n$. Corresponding adjustments to point coordinates are made as needed.

\section{(2) Stereographic Projection}

Theory and computations for $S$ are simplified by using stereographic projections. These projections map images of mathematical objects to and fro between $S$ and $E$. The projected objects and their images are called stereographic projection images of one another, or simply images. Typically $S$ or an object on $S$ is projected to $E$, where Möbius mappings are employed to transform the planar image of the object, and this transformed image is then projected back to $S$.

The spherical point $z=(x, y, t)^{\mathrm{T}}$ and the planar point $z_{\mathrm{E}}$ are images when $z$ and $z_{\mathrm{E}}$ are collinear with the fixed north pole $n$ of the sphere, and we write: $z \leftrightarrow z_{\mathrm{E}}$ or equivalently $z_{\mathrm{E}} \leftrightarrow z$. The improper point $\infty$ in $E$ and the north pole $n$ on $S$ are designated images ( $n \leftrightarrow \infty$ or $\infty \leftrightarrow n$ ), thus making stereographic projection a one-one and onto mapping between $S$ 
and $E$. It is useful to keep in mind the simple maxim that equal objects have equal images.

Let $P$ be the projection operator from $E$ to $S$. The image points of the image pair $\left(z \leftrightarrow z_{\mathrm{E}}\right)$ are expressed in terms of one another by

$$
\begin{aligned}
& \left\{z=P z_{\mathrm{E}}=\left(2 x_{\mathrm{E}} / r_{1}^{2}, 2 y_{\mathrm{E}} / r_{1}^{2},\left(r_{1}^{2}-2\right) / r_{1}^{2}\right)^{\mathrm{T}}\right\} \leftrightarrow \\
& \left\{z_{\mathrm{E}}=P^{-1} z=x /(1-t)+\mathrm{i} y /(1-t)\right\}, \text { or by } \\
& \left\{z_{\mathrm{S}}=(\theta, \varphi)\right\} \leftrightarrow\left\{z_{\mathrm{E}}=\cot \theta / 2 \exp (\mathrm{i} \varphi)\right\},
\end{aligned}
$$

where $\theta=2 \operatorname{acot} r, \cos \varphi=x_{\mathrm{E}} / r, \sin \varphi=y_{\mathrm{E}} / r, r_{1}{ }^{2}=r^{2}+1$ and $r^{2}=x_{\mathrm{E}}^{2}+y_{\mathrm{E}}^{2}$.

Now extend the scope of the image terminology and stereographic projection notation beyond points to other mathematical objects like functions, equations, regions and curves. Distortions inevitably occur in projected images, being small for objects near $s$ but violent for objects near $n$. However, the projection operators $P$ and $P^{-1}$ do have four important and undistorted features, here called the

Stereographic Projective Properties: one-one, onto, circle-preserving and improper.

Circle-preserving here means that (circles on $S) \leftrightarrow($ circles or straight lines in $E$ ). Improper (or anti-conformal) means isogonal or angle-preserving, in that two curves in $E$ intersect at the same angle as their images on $S$, but with opposite sense - one traversed $C C W$ (counter-clockwise), and the other $C W$ (clock-wise). And proper (or conformal) means isogonal but with the same sense. Proofs and numerous graphics pertaining to these four stereographic Projective Properties are provided by [1, pp. 140-148].

The Projective Properties are valid, where applicable, for the objects listed above: functions, regions, equations and curves. Circle-preserving is illustrated by: (center lines $L$ in $E) \leftrightarrow$ (great circles of longitude $C$ on $S$ ) and by (concentric circles about the origin of $E$ ) $\leftrightarrow$ (parallel circles of latitude on $S$ ). One-one and onto are then illustrated by $E=$ (union of the center lines of $E$ ) $=$ (union of the concentric circles about the origin of $E$ ), and $S=$ (union of the great circles of longitude $)=($ union of the parallel circles of latitude). Isogonality is evident in the orthogonal intersections of the center lines with the concentric circles about the origin in $E$, and the same orthogonal intersections in their respective images: the great circles of longitude orthogonal to the parallel circles of latitude on the sphere $S$. These examples show that (the familiar spherical coordinates $(\theta, \varphi)$ on $S) \leftrightarrow$ (the familiar polar coordinates $(\mathrm{r}, \varphi)$ in $E)$.

\section{(3) Planar Möbius Mappings}

Let $z_{\mathrm{E}}$ be a complex variable and $a^{\prime}$ and $b^{\prime}$ complex constants. If the three transformations $z_{\mathrm{E}} \rightarrow z_{\mathrm{E}}+a^{\prime}$ (shift in location), $z_{\mathrm{E}} \rightarrow b^{\prime} z_{\mathrm{E}}$ (change in scale/direction), and $z_{\mathrm{E}} \rightarrow 1 / z_{\mathrm{E}}$ (reciprocation) are compounded arbitrarily often and in arbitrary order, all the while allowing the constants $a^{\prime}$ and $b^{\prime}$ to vary from one transformation to the next, then the resulting function $w_{\mathrm{E}}$ of $z_{\mathrm{E}}$ is known as a homography or proper Möbius function (or proper function, for short), with the form

$$
w_{\mathrm{E}}=\left(a z_{\mathrm{E}}+b\right) /\left(c z_{\mathrm{E}}+d\right), \text { and we write: } w_{\mathrm{E}}=M_{\mathrm{E}} z_{\mathrm{E}},
$$

where $M_{\mathrm{E}}$ stands for the function. The coefficients $a, b, c, d$ are functions of the several values of $a^{\prime}$ and $b^{\prime}$. The function $w_{\mathrm{E}}$ $=M_{\mathrm{E}} z_{\mathrm{E}}$ is also called a Möbius transformation or Möbius mapping. It maps an extended complex plane to itself or to another extended complex plane in a one-one and onto manner. The special notation $z_{\mathrm{E}}^{\prime}=M_{\mathrm{E}} z_{\mathrm{E}}$ means that the $\mathrm{z}_{\mathrm{E}}$-plane is mapped to itself. If $z_{\mathrm{E}}$ is replaced by its complex conjugate $z_{\mathrm{E}} *$ in the function $w_{\mathrm{E}}=M_{\mathrm{E}} z_{\mathrm{E}}$, the resulting mapping is an anti-homography or improper Möbius function (improper, for short). A Möbius function with unspecified propriety is by default considered proper. The set of proper and improper Möbius mappings are surprisingly rich and versatile. They were used by [2] to model mean directions for circle-circle regression, and by [3] to model mean directions for spherical-spherical regression, while [4] used them to transform back and forth between circular Cauchy and linear Cauchy distributions, and to create compound transforms of circular and of linear Cauchy distributions. In this paper Möbius mappings are used for constructing the same kinds of operations on circular and spherical Cauchy distributions.

Every Möbius function $M_{\mathrm{E}}$ has associated with it the $2 \times 2$ matrix $M_{\mathrm{m}}$ of the complex coefficients $a, b, c, d$, with first row $(a, b)$ and second row $(c, d)$. For brevity this $2 \times 2$ matrix is written as

$M_{\mathrm{m}}=(a, b ; c, d)$,

where the m subscript of $M$ stands for a matrix, in contrast to the $E$ subscript used for planar points and mappings in $E$ or other extended complex planes. A Möbius matrix is always assumed to be of full rank two, otherwise the corresponding Möbius function degenerates to a constant.

The matrix $M_{\mathrm{m}}$ of a Möbius function $M_{\mathrm{E}}$ is not unique because any non-zero complex multiple $k M_{\mathrm{m}}$ of $M_{\mathrm{m}}$ is equivalent to $M_{\mathrm{m}}$ in that it yields the same value for $w_{\mathrm{E}}$ as does $M_{\mathrm{m}}$; these and related phenomena are collectively referred to as the $k$-effect. [1, pp. 148-158] gives an accounting of Möbius mappings using a geometric approach that is accompanied with illuminating graphics; see [5] for a more analytic but also insightful approach.

The set of all proper and improper Möbius mappings is a group under the operation of composition of mappings, and the compound mapping $M_{\mathrm{E} 2} M_{\mathrm{E} 1}$ is a Möbius mapping with matrix the product $M_{\mathrm{m} 2} M_{\mathrm{m} 1}$ of the corresponding Möbius matrices. This implies that the group of all proper Möbius mappings is homomorphic to the group of all nonsingular $2 \times 2$ complex matrices. See [1, pp. 148-158] for proofs. The subset of proper Möbius mappings is a subgroup, but the subset of improper mappings is not, because the composition of two improper mappings is a proper mapping.

Theorem 1 as stated below is a slightly modified adaptation of the fundamental theorem of Möbius mappings. A proof is given by [6, p. 46, pp. 106-109]. The Möbius Properties in this theorem deal with plane-to-plane mappings, and are similar to the Projective Properties above which pertain to sphere-plane mappings.

Theorem 1: (Fundamental theorem of Möbius mappings) A mapping $w_{\mathrm{E}}=M_{\mathrm{E}} z_{\mathrm{E}}$ from one extended complex $z_{\mathrm{E}}$-plane ( $z_{\mathrm{E}}{ }^{*}$-plane) to another or to itself is a proper (improper) Möbius mapping $\Leftrightarrow$ the mapping has the Möbius Properties: one-one, onto, circle-preserving and proper (improper). 
In plane-plane Möbius mappings circle-preserving means that circles or straight lines are mapped to circles or straight lines. Synonyms for proper mappings include homography, positive, conformal and rotational; synonyms for improper mappings include anti-homography, negative, anti-conformal and reflexive.

\section{(4) Spherical Images of Planar Möbius Mappings}

Spherical mappings are functions from the surface of one unit sphere to another or to itself. The spherical mapping $M$, $\mathrm{W}=M z$, and a planar Möbius mapping $M_{\mathrm{E}}, w_{\mathrm{E}}=M_{\mathrm{E}} z_{\mathrm{E}}$, are called images of one another, and we write $M \leftrightarrow M_{\mathrm{E}}$, when $\left(z \leftrightarrow z_{\mathrm{E}}\right) \Rightarrow\left(w \leftrightarrow w_{\mathrm{E}}\right)$ for all $z$ in $S$ and all $z_{\mathrm{E}}$ in $E$. In such case $M z=P\left(M_{\mathrm{E}} z_{\mathrm{E}}\right)=P M_{\mathrm{E}} P^{-1} z$ for all $z$, whence the equation

$$
M=P M_{\mathrm{E}} P^{-1} \text { and its inverse } M_{\mathrm{E}}=P^{-1} M P
$$

formally express the stereographic projection mappings between the spherical $M$ and planar Möbius $M_{\mathrm{E}}$ image functions. The one-one and onto Projection Properties of the projection operators $P$ and $P^{-1}$ ensure that the images $M$ and $M_{\mathrm{E}}$ are unique. A spherical mapping $M$ that is the image of some Möbius function $M_{\mathrm{E}}$ will be called a spherical Möbius mapping. A spherical Möbius mapping $M$ is proper or improper according as the argument of its image mapping is $z_{\mathrm{E}}$ or $z_{\mathrm{E}} *$. Equations (1) can be used to show the well-known morphic result that the spherical image of the composition $M_{\mathrm{E} 2} M_{\mathrm{E} 1}$ of two planar Möbius mappings is the composition of their corresponding spherical images, $M_{2} M_{1}$. This implies that the set of spherical Möbius mappings is isomorphic to the group of planar Möbius mappings, a conclusion which profoundly simplifies many statistical analyses and interpretations of spherical data. There is a spherical counterpart to the fundamental theorem of Möbius mappings:

Proposition 1: (Fundamental theorem of spherical Möbius mappings) A mapping $w=M z$ from one unit sphere to itself or to another unit sphere is a proper (improper) spherical Möbius mapping) $\Leftrightarrow M$ has the Möbius properties: one-one, onto, circle-preserving and proper (improper).

Proof: (proper, $\Rightarrow$ ) Assume that $M$ is a proper spherical Möbius mapping. Then $M$ is the image of a Möbius function $M_{\mathrm{E}}$, and $M$ inherits unscathed from $M_{\mathrm{E}}$ the three Projective Properties: one-one, onto, and circle preserving, so $M$ has all four proper Möbius Properties.

Proof: (improper, $\Rightarrow$ ) Same as above, with proper replaced therein by improper.

Proof: (proper, $\Leftarrow$ ) Assume $M$ has the proper Möbius Properties. Then the planar image of $M$, say $M_{\mathrm{E}}^{\prime}$, automatically retains the three common Möbius and Projective Properties: one-one, onto and circle-preserving, and is proper because of the even number of improper projections in (1). Then $M_{\mathrm{E}}^{\prime}$ is a proper Möbius function by the fundamental theorem of Möbius functions, $M$ is its image by (1), and $M$ is thus a spherical Möbius mapping by definition.

Proof: (improper, $\Leftarrow$ ) Assume that $M z$ has the improper Möbius Properties. We must show that its planar image, temporarily denoted by $M_{\mathrm{E}} w_{\mathrm{E}}$, say, is improper: $M_{\mathrm{E}} w_{\mathrm{E}}$ automatically inherits the one-one, onto and circle preserving projective properties, so we must show that $M_{\mathrm{E}} w_{\mathrm{E}}$ is improper. It has been shown by [3] that an improper spherical mapping $M z$ can be expressed as $M^{\prime} J z$ where $M^{\prime}$ is proper and $J=\operatorname{Diag}(1,-1,1)$. Then $M z=\left(M^{\prime} J\right) \mathrm{z}=M^{\prime}(J z)$. From the formulas in section 2.2 it is readily shown that $J z=z^{*}$ and $P z_{\mathrm{E}}{ }^{*}=\mathrm{z}^{*}$, where $\mathrm{z}^{*}=(\mathrm{x},-\mathrm{y}, \mathrm{z})^{\mathrm{T}}$ when $\mathrm{z}=(\mathrm{x}, \mathrm{y}, \mathrm{t})^{\mathrm{T}}$. Hence $M^{\prime} z^{*}=M z \leftrightarrow M_{\mathrm{E}} z_{\mathrm{E}}^{*}$, which implies $M_{\mathrm{E}} w_{\mathrm{E}}=M_{\mathrm{E}} z_{\mathrm{E}}{ }^{*}$ since the planar image is unique. This planar image of $M z$ is improper, and $M$ is therefore improper by definition.

\section{(5) Rescaling and Unitary Möbius Mappings}

The special planar Möbius rescaling mapping from $E$ to itself or to another extended complex plane is denoted by $\Delta_{\mathrm{E}}$, and defined by

$w_{\mathrm{E}}=\Delta_{\mathrm{E}} z_{\mathrm{E}}=\delta z_{\mathrm{E}}$,

with Möbius matrix $\Delta_{\mathrm{m}}=(\delta, 0: 0,1)$ where $\delta>0$. This proper mapping is the first of two special proper Möbius mappings used in this paper, and both mappings play key roles in all that follows.

Imagine the entire $z_{\mathrm{E}}$-plane rescaled as $z_{\mathrm{E}}^{\prime}=\delta z_{\mathrm{E}}, \delta>0$. The fixed points of this central planar dilation are 0 and $\infty$. Each center line $L$ of $E$ is mapped onto itself, and uniformly rescaled by $x_{\mathrm{L}}^{\prime}=\delta x_{\mathrm{L}}$ for each point $x_{\mathrm{L}}$ of each center line $L$. The concentric circles about the origin of $E$ are mapped into themselves as a whole. Each of the concentric circles intersects each center line orthogonally both before and after the rescaling, by the isogonality of Möbius mappings. Every concentric circle is altered unless the rescaler $\delta=1$, in which case none are altered. Rescaling uniformly moves the lines and circles of the planar polar coordinates toward the origin 0 , leaves them unchanged, or uniformly moves them away from 0 , according as the indicator function $\tau, \tau=\operatorname{sgn}(1-\delta)$, is 1,0 , or -1 .

The spherical image of the planar rescaling, $\left(z_{\mathrm{E}}^{\prime}=\delta z_{\mathrm{E}}\right)$, is the one-one mapping, $\Delta,\left(z^{\prime}=\Delta z\right.$ or $\left.S^{\prime}=\Delta S\right)$, of $S$ onto itself, where $z^{\prime}$ and $z$ on $S$ are the images of $\mathrm{z}_{\mathrm{E}}^{\prime}$ and $z_{\mathrm{E}}$ in $E$. This spherical Möbius mapping $\Delta$ operates as a nonlinear rescaling of the sphere by $\delta$. Its fixed points are $s$ and $n$, the images of the fixed points 0 and $\infty$ of its planar image $\Delta_{\mathrm{E}}$. The spherical image $(z=\Delta z)$ of the planar rescaling (2) is obtained in closed form via section 2.2 by substituting $\delta z_{\mathrm{E}}$ for $z_{\mathrm{E}}$ therein to get:

$z^{\prime}=\Delta z=\left(2 \delta x_{\mathrm{E}} / r_{\delta 1}^{2}, 2 \delta y_{\mathrm{E}} / r_{\delta 1}^{2},\left(r_{\delta 1}^{2}-2\right) / r_{\delta 1}^{2}\right)^{\mathrm{T}}$

for all $z^{\prime}$ on $S^{\prime}$, where the original $r_{1}{ }^{2}$ of section 2.2 is replaced by $r_{\delta 1}^{2}=\left(\delta x_{\mathrm{E}}\right)^{2}+\left(\delta y_{E}\right)^{2}+1$ to account for the switch to $\delta z$.

When the planar rescaling uniformly moves the lines and circles of the polar coordinates toward 0 , leaves them unchanged, or uniformly moves them away from 0 a mean $d i$ rection $\mu$ is induced on $S$ which is $\mu=s$, the south pole, or 0 (no mean direction), or $n$, the north pole, according as $\tau=1$, or 0 , or -1 . To capture quantitatively this directional phenomenon when $\Delta$ operates on $s$ we revise the definition of $\Delta s$ to

$\mu=\Delta s=\tau s=(s, 0, n)$ according as $\tau=(1,0,-1)$.

Equation (4) defines a new entity created by the rescaling: the creation of the mean spherical direction $\mu$ if $\tau \neq 0$, and the declaration of its non-existence: $\mu=0$, if $\tau=0$. 
Change of location mappings are the second of the two special Möbius mappings herein. A change of location mapping $A_{\mathrm{E}}$ has the form

$w_{\mathrm{E}}=A_{\mathrm{E}} z_{\mathrm{E}}=\left(\alpha z_{\mathrm{E}}+\beta\right) /\left(-\beta^{*} z_{\mathrm{E}}+\alpha^{*}\right)$,

whose matrix $A_{\mathrm{m}}=\left(\alpha, \beta ;-\beta^{*}, \alpha^{*}\right)$ is restricted by fixing its real-valued determinant, $\left|A_{\mathrm{m}}\right|=\alpha \alpha^{*}+\beta \beta^{*}$, to be unity. The matrix $A_{\mathrm{m}}$ is called a unitary matrix and its planar Möbius mapping $A_{\mathrm{E}}$ a unitary mapping. The set of all unitary mappings is, like the set of all change-of-scale mappings, a subgroup of the group of Möbius mappings, and thus is closed under composition of such mappings. Because of the restriction $\left|A_{\mathrm{m}}\right|=1$ on $A_{\mathrm{m}}$ the parametric degrees of freedom ( $p d f$, for short) for $A_{\mathrm{E}}$ and $A_{\mathrm{m}}$ is 3 , not 4 . The inverse of $A_{\mathrm{m}}$ is $A_{\mathrm{m}}{ }^{*}$ $=\left(\alpha^{*},-\beta ; \beta^{*}, \alpha\right)$, its conjugate transpose, which suggests (correctly) that a unitary matrix is the 2-dimensional complex counterpart of a real $2 \times 2$ proper rotation matrix.

The connection between the spherical image $(w=A z)$ of the planar Möbius unitary mapping $\left(w_{\mathrm{E}}=A_{\mathrm{E}} z_{\mathrm{E}}\right)$ in (5) was discovered by [7]. His remarkable result is summarized as follows:

Theorem 2: (Gauss' rotation image theorem): The spherical image of the proper unitary planar Möbius mapping $\left(w_{\mathrm{E}}\right.$ $\left.=A_{\mathrm{E}} z_{\mathrm{E}}\right)$ is the proper spatial rotation $(w=A z)$, where $(w, A, z)$ are the respective spherical images of $\left(w_{\mathrm{E}}, A_{\mathrm{E}}, z_{\mathrm{E}}\right)$ and $A$ is a real $3 \times 3$ proper rotation matrix. The spherical image of the improper unitary mapping $\left(w_{\mathrm{E}}=A_{\mathrm{E}} z_{\mathrm{E}}{ }^{*}\right)$ is the improper spatial rotation $(w=(A J) z)$, where $(w, A,(J z))$ are the respective images of $\left(w_{\mathrm{E}}, A_{\mathrm{E}}, z_{\mathrm{E}}{ }^{*}\right)$, and $J=\operatorname{Diag}(1,-1,1)$.

Any real $3 \times 3$ rotation matrix $A$ is proper, or improper, according as $|A|$ is +1 , or -1 . The use of $\mathrm{z}_{\mathrm{E}}{ }^{*}$ as the argument for Möbius functions is infrequent herein, but is included for completeness due to its potential importance. Details of Gauss' rotation image theorem for converting between complex unitary matrices and various forms of their real $3 \times 3$ rotation matrix images are in Appendix II.

\section{(6) Polar Decompositions}

For any Möbius matrix $M_{\mathrm{m}}$ the identity

$$
M_{\mathrm{m}} \equiv\left\{M_{\mathrm{m}}\left(M_{\mathrm{m}} * M_{\mathrm{m}}\right)^{-1 / 2}\right\} \times\left\{\left(M_{\mathrm{m}} * M_{\mathrm{m}}\right)^{1 / 2}\right\} \equiv A_{\mathrm{m}} \times G_{\mathrm{m}},
$$

uniquely defines the $2 \times 2$ unitary matrix $A_{\mathrm{m}}$ and the $2 \times 2$ positive hermitian matrix $G_{\mathrm{m}}$. Their matrix product $A_{\mathrm{m}} G_{\mathrm{m}}$ is called the polar decomposition (polar form, for short) of $M_{\mathrm{m}}$, and we write:

$$
M_{\mathrm{m}}=A_{\mathrm{m}} G_{\mathrm{m}} \text { (polar form). }
$$

If $M_{\mathrm{m}}$ is complex then $M_{\mathrm{m}} * M_{\mathrm{m}}$ is positive hermitian and can be decomposed into a principal form, and we write:

$$
G_{\mathrm{m}}^{2}=M_{\mathrm{m}} * M_{\mathrm{m}}=R_{\mathrm{m}} * \Delta_{\mathrm{m}}^{2} R_{\mathrm{m}} \text { (principal form) }
$$

where the matrix $R_{\mathrm{m}}$ is unitary and the columns of $R_{\mathrm{m}}{ }^{*}$ are the eigenvectors of $G^{2}$, and where $\Delta^{2}$ m is real and positive diagonal with its diagonal elements as the corresponding eigenvalues. The positive square root $G_{\mathrm{m}}=\left(M_{\mathrm{m}} * M_{\mathrm{m}}\right)^{1 / 2}$ of $G_{\mathrm{m}}^{2}$ is

$$
G_{\mathrm{m}}=R_{\mathrm{m}}^{*} \Delta_{\mathrm{m}} R_{\mathrm{m}} \text { (principal form) }
$$

where

$$
\Delta_{\mathrm{m}}=\operatorname{Diag}\left(\delta_{1}, \delta_{2}\right), \Delta_{\mathrm{m}}^{2}=\operatorname{Diag}\left(\delta_{1}^{2}, \delta_{2}^{2}\right),
$$

and both $\delta_{1}$ and $\delta_{2}$ are positive. The ratios $\delta=\delta_{1} / \delta_{2}$ and $\delta^{-1}$ are called rescalers for the Möbius matrix $M_{\mathrm{m}}$. The matrix $\Delta_{\mathrm{m}}$ is called the rescaling matrix. The unitary matrices $R^{*}{ }_{\mathrm{m}}$ and $R_{\mathrm{m}}$ are called rotors. If $\delta=1$ then $\Delta_{\mathrm{m}}$ is equivalent to the identity matrix $I_{2}$ because of the $k$-effect. This implies that $G_{\mathrm{m}}$ is also equivalent to $I_{2}$ and that $M_{\mathrm{m}}=A_{\mathrm{m}} \times \mathrm{I}_{2}$ (polar form).

Polar forms can be used to decompose any planar Möbius matrix into three unitary mappings and one rescaling mapping by combining the principal form of $G_{\mathrm{m}}$ and the polar form of $M_{\mathrm{m}}$ to get the extended polar form matrix expression:

$$
M_{\mathrm{m}}=A_{\mathrm{m}} R^{*}{ }_{\mathrm{m}} \Delta_{\mathrm{m}} R_{\mathrm{m}} \text { (extended polar form). }
$$

Complex Möbius matrices only have $p d f=6$ instead of 8 because of the $k$-effect. The $k$-effect must be accounted for when calculating the polar forms for Möbius matrices, otherwise $8 p d f$ will be foisted on a matrix which is only permitted 6 , and numerical errors will result. The $k$-effect can be accounted for by norming $M_{\mathrm{m}}$ (dividing each element of $M_{\mathrm{m}}$ by the square root of the determinant of $M_{\mathrm{m}}$ ). The resulting normed matrix is equivalent to $M_{\mathrm{m}}$ and has just $6 \mathrm{pdf}$. The extended polar form of a normed Möbius matrix will have the unitary matrices $A_{\mathrm{m}}, R_{\mathrm{m}}$ and $R^{*}{ }_{\mathrm{m}}$ of its polar form automatically normed by definition. The normed rescaling matrix must consequently have the form

$$
\Delta_{\mathrm{m}}=\operatorname{Diag}\left(\delta^{1 / 2}, \delta^{-1 / 2}\right) \text { or } \Delta_{\mathrm{m}}=\operatorname{Diag}\left(\delta^{-1 / 2}, \delta^{1 / 2}\right)
$$

where $\delta=\delta_{1} / \delta_{2}$ or $\delta_{2} / \delta_{1}$. This normed version is diagonal, is equivalent to the original $\Delta_{\mathrm{m}}$, has determinant equal to unity, and is unique for these three properties apart from the ordering of the eigenvalues (see section 3.2). Appendix I gives examples of matrix norming and polar form calculations.

The Möbius mapping $M_{\mathrm{E}}$ has an extended form similar to that of its Möbius matrix $M_{\mathrm{m}}$, and we write:

$$
M_{\mathrm{E}}=A_{\mathrm{E}} R_{\mathrm{E}}^{-1} \Delta_{\mathrm{E}} R_{\mathrm{E}} \text { (extended pseudo-polar form). }
$$

Spherical and planar Möbius mappings are functions, not matrices, and the spherical image $\Delta$ is non-linear. So, they do not have polar forms per se, hence the term pseudo-polar form above. For all practical purposes though, these pseudopolar forms are virtually identical to polar forms. See [3] for a proof and further details of:

Theorem 3: Any proper or improper spherical Möbius mapping $M$ can be expressed uniquely as $(M=A G=$ $A R_{\beta S} \Delta R_{\mathrm{S} \beta}$ ) (extended pseudo-polar form) with respective planar images $\left(M_{\mathrm{E}}=A_{\mathrm{E}} G_{\mathrm{E}}=A_{\mathrm{E}} R^{-1}{ }_{\mathrm{E} 0 \beta} \Delta_{\mathrm{E}} R_{\mathrm{E} 0 \beta}\right)$ (extended pseudo-polar form), where $A$ and $R_{\mathrm{S} \beta}$ are real $3 \times 3$ rotation matrices, and $R_{\mathrm{S} \beta}$ rotates a unit vector $\beta$ called the base, with spherical coordinates $\beta_{\mathrm{S}}=\left(\theta_{\beta}, \varphi_{\beta}\right)$, through an amount $\eta=\pi$ $-\theta_{\beta}$, to the south pole s along the meridian of the base $\beta$ and about a positive rotation axis $p$ with spherical coordinates $p_{\mathrm{S}}$ $=\left(\pi / 2, \varphi_{\beta}+\pi / 2\right)$. The mapping $M$ is proper, or improper, according as the determinant of the aligner $A$ is +1 , or -1 .

Theorem 3 describes the spherical rotor, $R_{\mathrm{S} \beta}$, as that proper rotation which rotates the base point $\beta$ to the south pole $s$ of $S$ along the $\varphi_{\beta}$-meridian of $\beta_{\mathrm{S}}$ (the shortest route), so that $\left(s=R_{\mathrm{S} \beta} \beta\right) \leftrightarrow\left(0=R_{\mathrm{E} 0 \beta} \beta_{\mathrm{E}}\right)$ : the planar image of $R_{\mathrm{S} \beta}$ sends $\beta_{\mathrm{E}}$ to 0 . The base $\beta$ is important to the implementation of (4) 
in the sequel, and constitutes the source for defining the mean direction $\mu$ on a sphere. It will be described in more detail later.

Unitary mappings and their spherical images have $p d f=3$ as a rule, but the definition of a rotor in Theorem 3 makes it possible to get the rotors $R_{\mathrm{S} \beta}$ or $R_{\mathrm{E} 0 \beta}$ as functions of either $\beta$, as given in theorem 3 , or of $\beta_{\mathrm{E}}=P^{-1} \beta$. Therefore any rotor has $p d f=2$, not 3 . Thus, if the base $\beta=(x, y, t)^{\mathrm{T}}$ is known then $\beta_{\mathrm{E}}=x /(1-t)+\mathrm{i} y /(1-t)$ from section 2.2. Since $R_{\mathrm{E} 0 \beta} \beta_{\mathrm{E}}=0$ then the first row of its matrix $R_{\mathrm{m} 0 \beta}$ must be proportional to $\left(1,-\beta_{\mathrm{E}}\right)$. By definition of a unitary matrix the second row of $R_{\mathrm{m} 0 \beta}$ is then proportional to $\left(\beta^{*} \mathrm{E}, 1\right)$, with the same constant of proportionality. Hence the matrix $Q_{\mathrm{m} 0 \beta}=\left(1,-\beta_{\mathrm{E}} ; \beta^{*} \mathrm{E}, 1\right)$ is equivalent to $R_{\mathrm{m} 0 \beta}$. We can get $R_{\mathrm{m} 0 \beta}$ by norming $Q_{\mathrm{m} 0 \beta}:\left|Q_{\mathrm{m} 0 \beta}\right|$ $=1+\left|\beta_{\mathrm{E}}\right|^{2}=D$, say, so that $R_{\mathrm{m} 0 \beta}=D^{-1 / 2} \times Q_{\mathrm{m} 0 \beta}$. Then we can get $R_{\mathrm{S} \beta}$ from $R_{\mathrm{m} 0 \beta}$ by using Gauss' theorem and the techniques in Appendix II.

The objects $A_{\mathrm{m}}, A_{\mathrm{E}}$, and $A$ are called aligners; $G_{\mathrm{m}}, G_{\mathrm{E}}$, and $G$ are called hermitian rescalers; $R_{\mathrm{m}}, R_{\mathrm{E} 0 \beta}$ and $R_{\mathrm{s} \beta}$ are called rotors, and $\Delta_{\mathrm{m}}, \Delta_{\mathrm{E}}, \Delta$ and $\delta$ are called rescalers. In theorem 3,s $=R_{\mathrm{S} \beta} \beta$ and the transpose of $R_{\mathrm{S} \beta}$, denoted by $R_{\beta \mathrm{S}}$, satisfies $\beta=R_{\beta S} \mathrm{~s}$. Then $\left(\beta=R_{\beta S} \mathrm{~s}\right) \leftrightarrow\left(\beta_{\mathrm{E}}=R_{\mathrm{E} \beta 0} 0\right)$, and the Möbius matrix for the mapping $R_{\mathrm{E} \beta 0}$ is $R_{\mathrm{m} \beta 0}$, the conjugate transpose of $R_{\mathrm{m} 0 \beta}$. In summary, we have

$M_{\mathrm{m}}=A_{\mathrm{m}} R_{\mathrm{m} \beta 0} \Delta_{\mathrm{m}} R_{\mathrm{m} 0 \beta}$ (extended polar form), for Möbius matrix domains,

$M_{\mathrm{E}}=A_{\mathrm{E}} R_{\mathrm{E} \beta 0} \Delta_{\mathrm{E}} R_{\mathrm{E} 0 \beta}$ (extended pseudo-polar form), for planar Möbius mapping domains,

$M=A R_{\beta \mathrm{S}} \Delta R_{\mathrm{S} \beta}$ (extended pseudo-polar form), for spherical Möbius mapping domains

as extensions of the polar form $M_{\mathrm{m}}=A_{\mathrm{m}} G_{\mathrm{m}}$ and pseudo-polar forms $M_{\mathrm{E}}=A_{\mathrm{E}} G_{\mathrm{E}}$ and $M=A G$. Theorem 3 and the extended forms above stem from the homomorphism between the Möbius mappings $M_{\mathrm{E}}$ and their Möbius matrices $M_{\mathrm{m}}$, and the isomorphism between the Möbius mappings $M_{\mathrm{E}}$ and their spherical Möbius mapping images $M$. The extended pseudopolar form for $M$ shows that any spherical Möbius mapping can be decomposed into three real $3 \times 3$ rotation matrices, namely: $A, R_{\beta S}$ and $R_{\mathrm{S} \beta}$ corresponding to spherical changes in location, and a nonlinear rescaler $\Delta$ which operates according to (3) as a nonlinear spherical change in scale. These extended polar and extended pseudo-polar forms provide frameworks for defining, constructing and performing transformations on the spherical Cauchy distributions in section 4 .

\section{(7) Basic Structures}

The mappings $M_{\mathrm{E}}$ and $M$ and the matrix $M_{\mathrm{m}}$ each have total $p d f=6$, not 8 , because of the $k$-effect. These are allocated as follows: $p d f=3$ for each corresponding aligner, $p d f=2$ for each base, and $p d f=1$ for their common rescaler $\delta$. Basic structures for the 3 domains of $M_{\mathrm{E}}, M$ and $M_{\mathrm{m}}$, are composed of the corresponding aligners, bases, and the rescaler. They determine each of $M_{\mathrm{m}}, M_{\mathrm{E}}$ and $M$ uniquely, and we write:

$$
\begin{aligned}
& M_{\mathrm{m}}=\left[A_{\mathrm{m}}, \beta_{\mathrm{E}}, \delta\right], \\
& M_{\mathrm{E}}=\left[A_{\mathrm{E}}, \beta_{\mathrm{E}}, \delta\right], \quad \text { (basic structures) } \\
& M=[A, \beta, \delta]
\end{aligned}
$$

to conveniently group the 3 basic structural parameters for each of the 3 object domains, and to emphasize the total dependency of the objects $M_{\mathrm{m}}, M_{\mathrm{E}}$ and $M$ on them. Any basic structure above is computable from $M_{\mathrm{m}}$. Indeed, each of the forms in this section can be computed from any of the three basic structures above using the methods and formulas in the two Appendices.

The spherical Möbius mapping $\mathrm{M}$ is proper or improper according as the mapping $\mathrm{MJ}$ is improper or proper. Also,

$M J=A R_{\beta \mathrm{S}} \Delta R_{\mathrm{S} \beta} J=(A J)\left(J R_{\beta \mathrm{S}} J\right)(J \Delta J)\left(J R_{\mathrm{S} \beta} J\right)$ (extended pseudo-polar form)

The two $J$ s surrounding the rescaler $\Delta$ disappear because the two operators $J$ and $\Delta$ commute and $J$ is equal to its own inverse, or involutory. The eigenvector rotor $J R_{\beta S} J$ sends $s$ to the base by definition, so the base is $J R_{\beta S} J s=J R_{\beta S} S=J \beta$. Therefore we have

$$
M J=[A J, J \beta, \delta] \text { (basic structure). }
$$

The basic structures above for $M J$ and for $M$ permit us to switch spherical Möbius mappings back and forth between proper and improper Möbius functions at will, and get the entire basic structure for either of them from that of the other.

\section{(8) Cauchy Distributions}

Many of the methods and results of this paper are natural extensions of a well-known and simple stereographic projection relating the standard linear Cauchy distribution on a line to the uniform distribution on a circle. Basic notation, definitions and selected results for linear and circular Cauchy distributions are set forth in this subsection for comparison and reference.

A Linear Cauchy distribution (LCD) with median $m$ and rescaler $\delta$ on a center line $L$ has the probability density function

$$
\mathrm{f}\left(x_{\mathrm{L}} ; m, \delta, L\right)=(\delta \pi)^{-1}\left[1+\left\{\left(x_{\mathrm{L}}-m\right) / \delta\right\}^{2}\right]^{-1}
$$

where $x_{\mathrm{L}}, m \in L,-\infty<x_{\mathrm{L}}, m<\infty$ and $\delta>0$. We say $x_{\mathrm{L}}$ is linear Cauchy $m, \delta$ on $L$, or $L$ is Cauchy $m, \delta$, and write: $x_{\mathrm{L}} \sim$ $\mathrm{LC}(m, \delta, L)$, or $L \sim \mathrm{LC}(m, \delta)$. The expected value of $x_{\mathrm{L}}$ does not exist. If the rescaler $\delta=0$ then $x_{\mathrm{L}}=m$ with probability 1 ; if $\delta=\infty$ then probability is uniformly distributed over $L$. The standard $\operatorname{LCD}$ is $\operatorname{LC}(0,1)$ and is the same as the t-distribution with one degree of freedom. Every LCD can be generated from the standard LCD by the linear transformation

$$
x_{\mathrm{L}} \sim \mathrm{LC}(0,1, L) \Leftrightarrow \delta x_{\mathrm{L}}+m \sim \mathrm{LC}(m, \delta, L), \delta>0 .
$$

Circular Cauchy distributions (CCDs) are dichotomized by their parametric form as being either classical (the current form) or rescaled (the new form introduced here). All the angular scale changes in this paper are results of projections: in classical forms the projection point is along the $\pm \mu$ axis and is either inside or outside $S$, while in rescaled forms the projection point is always the north pole $n$ on $\mathrm{S}$, which is conventionally $\mu$ or $-\mu$ when $\mu$ exists.

Classical and wrapped CCDs have the same probability density functions. Their common density, taken from [8, p. 51], was modified by using $\left|1-\rho^{2}\right|$, in place of $\left(1-\rho^{2}\right)$ in $[8$, p. 51], in the expressions for $A^{\prime}$ and $B^{\prime}$ just below the prob- 
ability density of the classical CCD in (6.2). The classical $\mathrm{CCD}$ is defined, for the moment, on the equator $U$ of $E$, and its density is:

$\mathrm{f}(z ; \zeta, U)=(2 \pi)^{-1}\left(A^{\prime}-B^{\prime} z^{\mathrm{T}} \mu\right)^{-1}$

where $z, \mu \in S$ but both are restricted to lie on $U$, and $A^{\prime}=$ $\left(1+\rho^{2}\right) /\left|1-\rho^{2}\right|>B^{\prime}=2 \rho /\left|1-\rho^{2}\right| \geq 0$. We say that $z$ is classical Cauchy $\zeta$ on $U$, or $U$ is classical Cauchy $\zeta$, and write: $z$ $\mathrm{CC}(\zeta, U)$, or $U \sim \mathrm{CC}(\zeta)$. The parameter $\zeta=r \mu$ is taken as complex when used in circle mappings, in which case $\mu$ is thought of as $\mu_{\mathrm{C}}=\exp (\mathrm{iv})$ in local coordinates, as per section 2.1. The classical CCD has $2 p d f$, one for the length, $r$, of $\zeta$, and the other for its direction $\mu$ which is constrained to lie on $\mathrm{U}$ and thus has $p d f=1$ only. The precision $\rho$ for $z$ is

$$
\rho=\min \left(r, r^{-1}\right), \rho \in[0,1),
$$

and the center of gravity is $\mathrm{E}(z ; \zeta, U)=\rho \mu$. If $\rho=1$ then $z=\mu$ with probability 1 ; if $\rho=0$ the distribution is uniform and written as $\mathrm{CC}(0, U)$ or $\mathrm{CC}(0)$. CCDs with the form (6.2) can be defined on any unit circle $C$ of $S$ containing $\pm \mu$ after appropriate interpretations of $z$ and $\mu$ as per section 2.1.

Circle mappings are defined, according to [6, p. 120122], as Möbius mappings which map one unit circle to itself or to another unit circle. Circle mappings were used by [2] to model the mean direction for circle-circle regression. [9] used the circle mapping model of [2] for the link function for a circular regression model, but replaced the von Mises distribution for regression deviations used by [2] with a classical CCD.

The most general circle mapping $M_{\mathrm{C}}$ has a matrix of the form $M_{\mathrm{m}}=\left(a, b ; b^{*}, a^{*}\right)$ where $|a|=1$ and $|b| \neq 1$, and so has $p d f=3$. See [6, p. 120-122] for a proof. A variety of forms for circle mappings can be created as reparameterizations or special cases of $M_{\mathrm{m}}$ (see e.g. [2] and [10]). Circle mappings with $|b|>1$ are improper. When $|b|<1$ they are proper. Circle mappings are closed under composition.

The special circle mapping $T_{\mathrm{C}}$ on a unit circle $C$, with $p d f$ $=2$, is expressed as

$\mathrm{z}^{\prime}=T_{\mathrm{C}} z=\left\{(z-\zeta) /\left(\zeta^{*} z-1\right)\right\}$,

where $T_{\mathrm{C}}$ has matrix $T_{\mathrm{m}}=\left(1,-\zeta ; \zeta^{*},-1\right), r=|\xi| \neq 1$, and the point $z$ is constrained to lie on $C$. The group property of circle mappings makes them useful for modifying CCDs, and [4]s circle mapping $T_{\mathrm{C}}$ is used extensively for that purpose in this paper. Using (6.3), [4] derives:

Theorem 4: $C \sim \mathrm{CC}\left(\zeta_{1}\right) \Leftrightarrow T_{\mathrm{C}} C \sim \mathrm{CC}\left(T_{\mathrm{C}} \zeta_{1}\right)$,

where $T_{\mathrm{C}} \zeta_{1}\left(\zeta_{1}-\zeta\right) /\left(\zeta^{*} \zeta_{1}-1\right)$, an expression that is akin to relativistic addition of velocities. The demonstration that the complex parameter for $T_{\mathrm{C}} C$ is $T_{\mathrm{C}} \zeta_{1}$ depends on a harmonic property of CCDs (see [4] or [8, p. 52]).

If $\zeta_{1}=0$ then $T_{\mathrm{C}} \zeta_{1}=\zeta$. If $\zeta=\zeta_{1}$ then $T_{\mathrm{C}} \zeta_{1}=0$, implying a uniform distribution, which in turn implies that $T_{\mathrm{C}}$ is involutory (equivalent to its own inverse). It is readily shown that a Möbius matrix is involutory if and only if its trace is zero. An equivalent inverse to any Möbius matrix $(a, b ; c, d)$ is $(d,-$ $b ;-c, a)$, obtained by swapping $a$ and $d$ and changing the signs of $b$ and $c$.

Rescaled CCDs are defined, like classical CCDs, on any great circle $C$ of $S$ that contains $\mu$. The rescaled CCD and classical CCD have the same circular Cauchy distribution, being merely different parameterizations of that same distribution. The classical CCD has two parameters: $(\mu, r)$ or $(\mu, \rho)$, with $\xi=r \mu$ complex, and the rescaled CCD has two parameters: $(\mu, \delta)$. The two CCD forms are easily differentiated by their notations, $r$ or $\rho$ vs. $\delta$, and consequently the form need not be explicitly stated when describing a particular CCD. The rescaled CCD has probability density function

$\mathrm{f}(z ; \mu, \delta, C)=(2 \pi)^{-1}\left(A-\tau B z^{\mathrm{T}} \mu\right)^{-1}$

where $z \in S$ but is restricted to $C, \pm \mu \in C$ when $\delta \neq 1, \delta>0$, $A=\left(1+\delta^{2}\right) /(2 \delta)>\tau B=\left\{\tau\left(1-\delta^{2}\right)\right\} /(2 \delta) \geq 0$, and the indicator function $\tau$ is defined, as before, by

$\tau=\operatorname{sgn}(1-\delta)=(1,0,-1)$ according as $\delta$ is $(<1,=1,>1)$.

The requirements that $A>\tau B \geq 0$ in (6.4) and $A^{\prime}>B^{\prime} \geq 0$ in (6.2) serve to avoid singularities and force the densities to be maximized at $z=\mu$. We say that $z$ is rescaled circular Cauchy $\mu, \delta$ on $C$, or $C$ is rescaled Cauchy $\mu, \delta$, and write: $z$ $\sim \mathrm{CC}(\mu, \delta, C)$, or $C \sim \mathrm{CC}(\mu, \delta)$. If $\delta=0$ or $\infty$ then $z=\mu$ with probability 1 . The closer $\delta$ is to unity the greater is the scatter of the observations about $\mu$. The rescaled CCD is uniform $\Leftrightarrow$ $\delta=1$ or equivalently $\Leftrightarrow \tau=0$, in which case the mean direction does not exist and we write $C \sim \mathrm{CC}(0,1)$. The inference that $\mu=0$ (which is an impossible value) in this $\operatorname{CC}(0,1)$ notation is intended to indicate that $\mu$ does not exist, and we use $\mathrm{CC}(0,1)$ or $\mathrm{CC}(0,1, C)$ to indicate that the $\mathrm{CCD}$ is uniform, for notational simplicity and conformity with (4) and the indicator function $\tau$. The density (6.4) for the rescaled CCD is derived in section 3 .

Spherical Cauchy distributions (SCDs) are derived in section 4. They are labeled, like CCDs, as either classical or rescaled. The classical SCD is constructed from the classical CCD of (6.2), and it is convenient to assign the same parameters $(\mu, r)$ or $(\mu, \rho)$ or $\xi=r \mu$ or $\xi=\rho \mu$ for the classical SCD as for the classical CCD from which it was derived. Likewise, the rescaled SCD is constructed from the rescaled CCD of (6.4), and it has the same parameters $(\mu, \delta)$ as the rescaled CCD from which it was derived. Further details regarding spherical Cauchy distributions are in section 4 .

\section{CIRCULAR CAUCHY DISTRIBUTIONS}

\section{(1) Get Rescaled CCD From Uniform CCD}

Proposition 2 below quantifies the manner in which the mean direction and scatter on any rescaled Cauchy great circle $C$ of longitude through $\pm s$ on $S$ are influenced by the rescaler $\delta$. This result is central to all that follows.

Proposition 2: (a) For any great circle $C$ of longitude on $S$ and any center line $L$ in $E, C \leftrightarrow L \Rightarrow C \sim \mathrm{CC}(\tau \mathrm{s}, \delta) \Leftrightarrow L \sim$ $\operatorname{LC}(0, \delta)\}$.

(b) If $S$ is uniform then the rescaling $\Delta S$ of $S$ by $\delta$ induces identical rescaled $\mathrm{CC}(\tau \mathrm{s}, \delta)$ distributions on each great circle of longitude $C$ on $S$.

(c) For given $\tau s$, rotating $\Delta S$ of (b) by the Rotor $R_{\mu \tau \mathrm{S}}(\mu=$ $\left.R_{\mu \tau \mathrm{S}} \tau \mathrm{S}\right)$ sends the mean $\tau s$ to the new mean $\mu$, and sends the great circles $C$ through $\pm \tau s$, with identical rescaled $\mathrm{CC}(\tau \mathrm{s}, \delta)$ distributions, to the great circles $R_{\mu \mathrm{rS}} C$ through $\pm \mu$, with identical rescaled $\mathrm{CC}(\mu, \delta)$ distributions. 
The proof of Proposition 2 is carried out in steps, the first of which is the proof of the well-known result alluded to at the beginning of section 2.8 which relates the standard LCD on a center line $L$ in $E$ to the uniform distribution on a great circle of longitude $C$ on $S$. This well-known result is a special case of (a) in Proposition 2 and may be expressed symbolically as

$$
C \leftrightarrow L \Rightarrow\{C \sim \mathrm{CC}(0,1) \Leftrightarrow L \sim \mathrm{LC}(0,1)\} .
$$

If $C \leftrightarrow L$ then $C$ and $L$ are coplanar with common east longitude $\varphi$, say, which locates $C$ and $L$. All subsequent activity pertaining to (7) is limited to points either on $C$ or on $L$, so the local variables $\gamma \in C$ and $x_{\mathrm{L}} \in L$ identified with such points suffice for the proof of (7), and their common longitude $\varphi$ can be ignored. It follows that the proof of (7) given below applies to every $C \leftrightarrow L$ image pair of $S$ and $E$.

Proof of (7): $(\Rightarrow)$ Assume $C \sim \mathrm{CC}(0,1)$. The uniform density for $\gamma$ is $\mathrm{f}(\gamma ; C)=(2 \pi)^{-1}$. Fig. (1) is a planar crosssection of $S$ and $E$ showing the plane of the great circle of longitude $C$ and its center line image $L$. The dashed lines in the Figure are used later. From Fig. (1) the point $\gamma$ (with origin $s$ ) on $C$ represents the central angle $\gamma=\angle(\gamma, 0, s)$, since $\gamma$ is measured $\mathrm{CCW}$ from $s$.

The central angles $\gamma$ and $\angle(n, 0, \gamma)$ are supplementary, and triangle $(n, 0, \gamma)$ is isosceles, so $\gamma=2 \beta$ where $\beta=\angle(n, \gamma, 0)$ $=\angle 0, n, \gamma)$. The points $n$ and $\gamma$ on $C$ and $x_{\mathrm{L}}$ on $L$ are collinear, so $\gamma \leftrightarrow x_{\mathrm{L}}$. Triangle $\left(n, 0, x_{\mathrm{L}}\right)$ is right, so $x_{\mathrm{L}}=$ opposite/adjacent $=\tan \beta=\tan \gamma / 2$, a one-one mapping from $C$ onto $L$ which shows the functional relationship characterizing the $C \leftrightarrow L$ image pair. The density this induces on $L$ is LC $(0,1)$, the standard LCD of (6.1), since

$$
\begin{gathered}
\mathrm{f}\left(x_{\mathrm{L}} ; L\right)=\mathrm{f}(\gamma ; C)\left|\mathrm{d} \gamma / \mathrm{d} x_{\mathrm{L}}\right|=(2 \pi)^{-1}\left|\left(2 \cos ^{2} \gamma / 2\right)\right| \\
=(\pi)^{-1}\left|1+\tan ^{2} \gamma / 2\right|^{-1}=(\pi)^{-1}\left(1+x_{\mathrm{L}}^{2}\right)^{-1} .
\end{gathered}
$$

$(\Leftarrow)$ The converse is shown by repeating the $(\Rightarrow)$ argument above with the roles of $\gamma$ and $x_{\mathrm{L}}$ reversed. This completes the proof of (7).

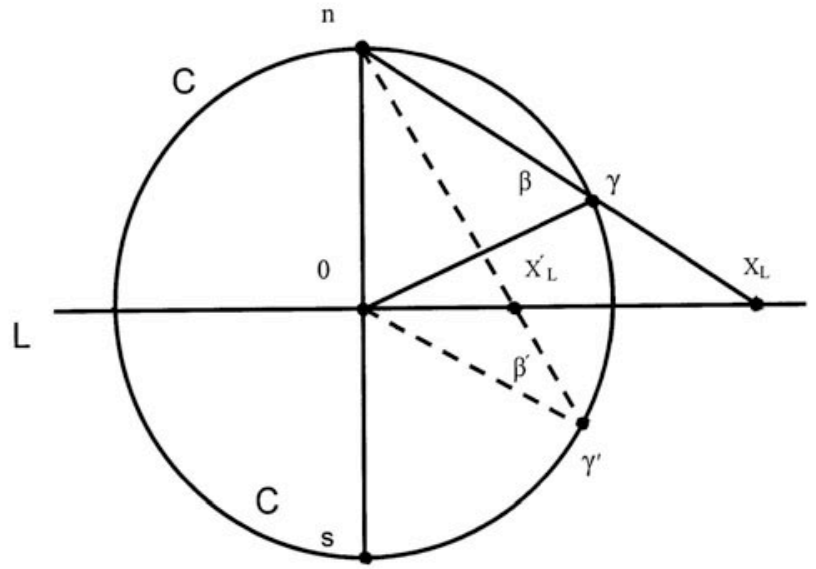

Fig. (1). Geometry of Rescaled Directional Cauchy Distributions.

Proof of Proposition 2(a): Assume $C \leftrightarrow L$ for some east longitude $\varphi$. As in the proof of (7), the local variables $\gamma$ and $x_{\mathrm{L}}$ suffice for the analysis, and they are independent of $\varphi$. The mapping $x_{\mathrm{L}}=\tan \gamma / 2$ is one-one and onto the center line $L$. If $\delta=1$ there is no rescaling and the proposition reduces to (7), consistent with the notation $\mu=0$ for the uniform case, and $C$ is uniform. Assume that $\delta \neq 1$.

$(\Leftarrow)$ Instead of assuming directly that the center line $L \sim$ $\operatorname{LC}(0, \delta)$, it is convenient to start with the weaker assumption that $x_{\mathrm{L}} \sim \mathrm{LC}(0,1, L)$, as in (7). This implies that $\gamma \sim \mathrm{CC}(0,1, C)$ by (7) and further implies, via section 2.8 , that $x_{\mathrm{L}}^{\prime}=\delta x_{\mathrm{L}} \sim$ $\mathrm{LC}(0, \delta, L)$. We will show that the circular image $\gamma^{\prime}$ on $C$ of the rescaled point $x_{\mathrm{L}}^{\prime}$ on $L$ is $\mathrm{CC}(\tau \mathrm{s}, \delta, C)$, (Fig. $\left.\mathbf{1}\right)$, by showing that the density of $\gamma^{\prime}$, given by

$$
\mathrm{f}\left(\gamma^{\prime} ; \tau \mathrm{s}, \delta, C\right)=\mathrm{f}(\gamma ; 0,1, C)\left|\mathrm{d} \gamma / \mathrm{d} \gamma^{\prime}\right|
$$

has the form (6.4). The angular point $\gamma^{\prime}$ (the rescaled $\gamma$ on $C$ in Fig. 1) represents the central angle $\gamma^{\prime}=\angle\left(\gamma^{\prime}, 0, s\right)$. Both $\gamma^{\prime}$ and $\gamma$ must be measured as angular deviations from $\mu=\tau s$ when $\tau \neq 0$, as per the convention at the end of section 2.1. The circle $C$, indeed, the entire sphere $S$, has been rescaled using $\delta \approx 2 / 5$ in Fig. (1), so $\tau=1$ and $\mu=s$ : the points $\gamma^{\prime}$ on $C$ and $x_{\mathrm{L}}^{\prime}$ on $L$ and angles $\left(n, 0, \gamma^{\prime}\right)$ and $\left(0, n, \gamma^{\prime}\right)$, after the rescaling has been done, correspond to the configuration of the original points $\gamma$ on $C, x_{\mathrm{L}}$ on $L$ and angles $(n, 0, \gamma)$ and $(0, n, \gamma)$ before the rescaling. Get $x_{\mathrm{L}}^{\prime}=\delta x_{\mathrm{L}}$ and plot it on $L$. Draw the line through $n$ and $x_{\mathrm{L}}^{\prime}$, extended to meet $C$ at $\gamma^{\prime}$, so that $\gamma^{\prime} \leftrightarrow$ $x_{\mathrm{L}}^{\prime}$. Triangle $\left(n, 0, \gamma^{\prime}\right)$ is isosceles and triangle $\left(\mathrm{n}, 0, x_{\mathrm{L}}^{\prime}\right)$ is right, so that, as in the proof of (7), $x_{\mathrm{L}}^{\prime}=\mathrm{opp} / \mathrm{adj}=\tan \gamma^{\prime} / 2$, a oneone mapping from $C$ onto $L$.

Then $\tan \gamma^{\prime} / 2=x_{\mathrm{L}}^{\prime}=\delta \tan \gamma / 2$. Differentiate $x_{\mathrm{L}}^{\prime}$ as follows to get the Jacobian $\left|\mathrm{d} \gamma / \mathrm{d} \gamma^{\prime}\right|$, and thereby the density for $\gamma^{\prime}$ on C:

$$
\mathrm{d} x_{\mathrm{L}}^{\prime}=\mathrm{d} \tan \gamma^{\prime} / 2=\sec ^{2} \gamma^{\prime} / 2 \mathrm{~d} \gamma^{\prime} / 2=\left(1+\tan ^{2} \gamma^{\prime} / 2\right) \mathrm{d} \gamma^{\prime} / 2,
$$

and also

$$
\mathrm{dx}_{\mathrm{L}}^{\prime}=\delta \mathrm{d} \tan \gamma / 2=\delta \sec ^{2} \gamma / 2 \mathrm{~d} \gamma / 2=\delta\left(1+\tan ^{2} \gamma / 2\right) \mathrm{d} \gamma / 2 .
$$

Next, equate the two expressions for $\mathrm{d} x_{\mathrm{L}}^{\prime}$, solve for $\mathrm{d} \gamma / \mathrm{d} \gamma^{\prime}$, then use successively the substitutions:

$$
\tan ^{2} \gamma / 2 \rightarrow \delta^{-2} \tan ^{2} \gamma^{\prime} / 2, \tan ^{2} \gamma^{\prime} / 2 \rightarrow \sin ^{2} \gamma^{\prime} / 2 / \cos ^{2} \gamma^{\prime} / 2
$$

followed by multiplication of numerator and denominator by $\cos ^{2} \gamma^{\prime} / 2$, then use the substitutions

$\cos ^{2} \gamma^{\prime} / 2+\sin ^{2} \gamma^{\prime} / 2 \rightarrow 1, \cos ^{2} \gamma^{\prime} / 2 \rightarrow 1 / 2\left(1+\cos \gamma^{\prime}\right)$, and $\sin ^{2}$ $\gamma^{\prime} / 2 \rightarrow^{1 / 2}\left(1-\cos \gamma^{\prime}\right)$ to simplify $\mathrm{d} \gamma / \mathrm{d} \gamma^{\prime}$, getting, in turn,

$$
\begin{aligned}
& \mathrm{d} \gamma / \mathrm{d} \gamma^{\prime}=\left(1+\tan ^{2} \gamma^{\prime} / 2\right) /\left\{\delta\left(1+\tan ^{2} \gamma / 2\right)\right. \\
& =\left(1+\tan ^{2} \gamma^{\prime} / 2\right) /\left\{\delta\left(1+\delta^{-2} \tan ^{2} \gamma^{\prime} / 2\right)\right\} \\
& =1 /\left(\delta \cos ^{2} \gamma^{\prime} / 2+\delta^{-1} \sin ^{2} \gamma^{\prime} / 2\right) \\
& =1 /\left\{\delta^{2}\left(1+\cos \gamma^{\prime}\right) /(2 \delta)+\left(\left(1-\cos \gamma^{\prime}\right) /(2 \delta)\right\}\right. \\
& =1 /\left[\left\{\left(\delta^{2}+1\right) /(2 \delta)\right\}-\left\{\left(1-\delta^{2}\right) /(2 \delta)\right\} \cos \gamma^{\prime}\right] \\
& =\left[A-B \cos \gamma^{\prime}\right]^{-1}
\end{aligned}
$$

say, where $z, \mu \in C, \cos \gamma^{\prime}=z^{\mathrm{T}} \mu$, and $A=\left(\delta^{2}+1\right) /(2 \delta)>B=$ $\left(1-\delta^{2}\right) /(2 \delta)>0$. For Fig. (1), $\delta \approx 2 / 5<1$, so $\tau=1$ and $\mu=s$. If $\delta$ had been greater than 1 we would have gotten the same symbolic result: $\left[A-B \cos \gamma^{\prime}\right]^{-1}$, but with $B<0$ and $\mu=\tau s=$ $n$, not $s$. Further, the deviation from $\mu$ would be the supplement $\pi-\gamma^{\prime}$ of $\gamma^{\prime}$, not $\gamma^{\prime}$, because $\mu$ would be $n$, not $s$. However, since $\cos \left(\pi-\gamma^{\prime}\right)=-\cos \gamma^{\prime}$ all these potential discrepancies disappear on replacing $B \cos \gamma^{\prime}$ with the equivalent but adaptive expression $(\tau B) z^{\mathrm{T}}(\tau s)$ where $\tau=\operatorname{sgn}(1-\delta)$. Then, on putting $\mu=\tau$ s we get 
$\mathrm{f}\left(\gamma^{\prime} ; \tau s, \delta, C\right)=(2 \pi)^{-1}\left\{A-\tau B z^{\mathrm{T}} \mu\right\}^{-1}$.

which is the same as (6.4). This adroitly morphs to the proper density for $\gamma^{\prime}$ when $C$ is any great circle of longitude on $\mathrm{S}, z \in C$, and $\delta>0$. Moreover, $z^{\mathrm{T}}(\tau s)=\cos \gamma^{\prime}$ and $A=$ $\left(1+\delta^{2}\right) /(2 \delta)>\tau B=\left\{\tau\left(1-\delta^{2}\right)\right\} /(2 \delta) \geq 0$, preserving all the requirements: $A>B \geq 0$, with $\gamma$ and $\gamma^{\prime}$ measured as angular deviations from $\mu=\tau s$.

$\Leftrightarrow$ Before rescaling, $x_{\mathrm{L}}=\tan \gamma / 2 \sim \mathrm{LC}(0,1, C)$, and $\mathrm{f}\left(\mathrm{x}_{\mathrm{L}}\right.$; $0,1, C)=(\pi)^{-1}\left(1+x^{2}\right)^{-1}$. After rescaling $C, x_{\mathrm{L}}^{\prime}=\delta x_{\mathrm{L}}$, and $\mathrm{f}\left(x_{\mathrm{L}}^{\prime} ; \quad 0, \delta, C\right)=\mathrm{f}\left(x_{\mathrm{L}} ; \quad 0,1, C\right) / \delta=(\pi \delta)^{-1}\left(1+\mathrm{x}_{\mathrm{L}}^{2}\right)^{-1}=$ $(\pi \delta)^{-1}\left\{1+\left(\mathrm{x}_{\mathrm{L}}^{\prime} / \delta\right)^{2}\right\}^{-1}$, so that $x_{\mathrm{L}}^{\prime} \sim \mathrm{LC}(0, \delta, C)$ by $(6.1)$.

Proof (b) and (c): The density (8) is independent of the common longitude $\varphi$ for $C$ and $L$ which, together with remarks made in and after the proof of (7), prove part (b). Part (c) follows because the rotor $R_{\mu \tau \mathrm{s}}$ rotates the mean direction $\tau$ s to $\mu$ and a circle $C$ to $R_{\mu \tau} C$, but does not affect the scatter and thus does not affect $\delta$.

\section{(2) Dualities in CCDS}

There is a duality in the rescaled $\mathrm{CC}(\mu, \delta, C)$ density (6.4): it is equivalent to a $\mathrm{CC}\left(-\mu, \delta^{-1}, C\right)$ density. To show this, assuming $\delta<1$, emphasize that $A$ and $B$ are functions of $\delta$ by putting $A(\delta)=\left(1+\delta^{2}\right) /(2 \delta)$ and $\tau B(\delta)=\left(1-\delta^{2}\right) /(2 \delta)$, and putting $A\left(\delta^{-1}\right)$ and $B\left(\delta^{-1}\right)$ as the corresponding functions of $\delta^{-1}$. Then, substituting in (8),

$\mathrm{f}\left(\gamma^{\prime} ; \mu, \delta, C\right) \propto\left[A(\delta)-\tau B(\delta) z^{\mathrm{T}} \mu\right]^{-1}$, with $A(\delta)>B(\delta) \geq 0$,

while, with the same proportionality constant,

$\mathrm{f}\left(\gamma^{\prime} ;-\mu, \delta^{-1}, C\right) \propto\left[A\left(\delta^{-1}\right)-B\left(\delta^{-1}\right) z^{\mathrm{T}}(-\mu)\right]^{-1}$

$=\left[A(\delta)-B(\delta) z^{\mathrm{T}} \mu\right]^{-1} \propto \mathrm{f}\left(\gamma^{\prime} ; \mu, \delta, C\right)$.

An analogous argument is used when $\delta>1$. This duality results from unavoidable ambiguities in the definition of the rescaler $\delta$ in section 2.6. To explain this, let $M_{\mathrm{E}}$ be any Möbius mapping whose rescaler $\delta \neq 1$, and let its matrix $M_{\mathrm{m}}$ have polar form $A_{\mathrm{m}} G_{\mathrm{m}}$, where $G_{\mathrm{m}}^{2}=M_{\mathrm{m}}{ }^{*} M_{\mathrm{m}}=R_{\mathrm{m} \beta 0} \Delta_{\mathrm{m}}^{2} R_{\mathrm{m} 0 \beta}$ (principal form). Let $R_{\mathrm{m} \beta 0}$ be partitioned as $R_{\mathrm{m} \beta 0}=\left(C_{1}, C_{2}\right)$, where $C_{1}$ and $C_{2}$ are the eigenvectors of $G_{\mathrm{m}}$, and $\Delta_{\mathrm{m}}=$ $\operatorname{Diag}\left(\delta_{1}, \delta_{2}\right)$ the corresponding eigenvalues. Then the conjugate transpose $\mathrm{R}_{\mathrm{m} 0 \beta}=\left(\mathrm{C}_{1}, \mathrm{C}_{2}\right)^{*}$ and

$$
G_{\mathrm{m}}=\left(\mathrm{C}_{1}, \mathrm{C}_{2}\right) \operatorname{Diag}\left(\delta_{1}, \delta_{2}\right)\left(\mathrm{C}_{1}, \mathrm{C}_{2}\right)^{*}=\delta_{1} \mathrm{C}_{1} \mathrm{C}_{1} *+\delta_{2} \mathrm{C}_{2} \mathrm{C}_{2} * \text {, }
$$

which can also be written as

$$
\mathrm{G}_{\mathrm{m}}=\delta_{2} \mathrm{C}_{2} \mathrm{C}_{2} *+\delta_{1} \mathrm{C}_{1} \mathrm{C}_{1} *=\left(\mathrm{C}_{2}, \mathrm{C}_{1}\right) \operatorname{Diag}\left(\delta_{2}, \delta_{1}\right)\left(\mathrm{C}_{2}, \mathrm{C}_{1}\right)^{*} \text {, }
$$

in effect swapping the positions of $\mathrm{C}_{1}$ and $\mathrm{C}_{2}$ and also those of $\delta_{1}$ and $\delta_{2}$. This ambiguity brings about the duality.

In the $\delta_{1}, \delta_{2}$ swap the rescaler for $\mathrm{G}_{\mathrm{m}}$ changes from $\delta=$ $\delta_{1} / \delta_{2}$ to $\delta^{-1}=\delta_{2} / \delta_{1}$, and either of these is just as legitimate as the other.

Before the $\mathrm{C}_{1}, \mathrm{C}_{2}$ column swap we had $R_{\mathrm{mo \beta}} \beta_{\mathrm{E}}=0$ and $R_{\text {mo } \beta}\left(-1 / \beta_{\mathrm{E}}{ }^{*}\right)=\infty$, with corresponding spherical images $R_{0 \beta} \beta$ $=s$ and $R_{0 \beta}(-\beta)=n$. After the $\mathrm{C}_{1}-\mathrm{C}_{2}$ swap, $\mathrm{R}_{\mathrm{m} 0 \beta}$ and $\mathrm{R}_{0 \beta}$ become, say, $\mathrm{R}_{\mathrm{m} 0 \beta}^{\prime}$ and $\mathrm{R}_{0 \beta}^{\prime}$. Then $R_{\mathrm{m} 0 \beta}^{\prime} \beta_{\mathrm{E}}=\infty$ and $\mathrm{R}_{\mathrm{m} 0 \beta}\left(-1 / \beta_{\mathrm{E}}\right)$ $=0$, with corresponding spherical images $R_{0 \beta}^{\prime} \beta=-s$ and $R_{0 \beta}^{\prime}(-\beta)=-n$, and this eigenvector swap causes a change in sign of $\mu$, which is just as legitimate a mean direction as the original $\mu$. Therefore the pairs $(\mu, \delta)$ and $\left(-\mu, \delta^{-1}\right)$ are dual sets of parameters.
To illustrate these results we will find the rescalers for the general circle mapping $M_{\mathrm{C}}$ with the patterned matrix $M_{\mathrm{m}}$ $=\left(a, b ; b^{*}, a^{*}\right)$ where $|\mathrm{a}|=1$ and $|b|=r \neq 1$. Then

$$
G_{\mathrm{m}}^{2}=M_{\mathrm{m}}{ }^{*} M_{\mathrm{m}}=R_{\mathrm{m}}{ }^{*} \Delta_{\mathrm{m}}^{2} R_{\mathrm{m}}=\left(1+r^{2}, 2 a^{*} b ; 2 a b^{*}, 1+r^{2}\right) .
$$

The eigenvalues $\delta_{1}{ }^{2}, \delta_{2}{ }^{2}$ say, of the positive hermitian matrix $G^{2}$ are the solutions of the equations

$$
\begin{aligned}
& \operatorname{tr} G^{2}{ }_{\mathrm{m}}=\operatorname{tr} \Delta_{\mathrm{m}}^{2}=\delta_{1}{ }^{2}+\delta_{2}{ }^{2}=2\left(1+r^{2}\right) \text { and } \\
& \left|G_{\mathrm{m}}^{2}\right|=\left|\Delta_{\mathrm{m}}{ }_{\mathrm{m}}\right|=\delta_{1}{ }^{2} \delta_{2}{ }^{2}=\left(1+r^{2}\right)^{2}-4 r^{2}=\left(1-r^{2}\right)^{2} .
\end{aligned}
$$

Eliminating $\delta_{1}^{2}$, the solutions are found to be the roots of a quadratic equation in $\lambda^{2}$, say; that is, the roots in $\lambda^{2}$ of

$$
\lambda^{4}-2\left(1+r^{2}\right) \lambda^{2}+\left(1-r^{2}\right)^{2}=0,
$$

namely: $\lambda^{2}=(1 \pm r)^{2}$ for the eigenvalues of $G_{\mathrm{m}}^{2}$. The eigenvalues $\delta_{1}$ and $\delta_{2}$ of $G_{\mathrm{m}}$ must both be positive since $G_{\mathrm{m}}$ is positive hermitian, so that $\delta_{1}=1+r$ and $\delta_{2}=|1-r|$, say. The rescalers of $\mathrm{G}_{\mathrm{m}}$ are therefore the ratios

$$
\delta=\delta_{1} / \delta_{2}=(1+r) /|1-r| \text { and } \delta^{-1}=\delta_{2} / \delta_{1}=|1-r| /(1+r) .
$$

The classical $\operatorname{CC}(\rho, \mu, C)$ density (6.2) has a duality also, namely $\mathrm{f}\left(\gamma^{\prime} ; \rho \mu, C\right)=\mathrm{f}\left(\gamma^{\prime} ; \rho^{-1} \mu, C\right)$. This was used by [4] to elegantly describe CCDs induced by applying Möbius mappings to LCDs or CCDs. This technique would not have worked if the customary formulation by $[8, \mathrm{p} .51]$ of the circular Cauchy density had not been revised to give (6.2).

\section{(3) Equivalence of Classical and Rescaled CCDs}

The disparate classical and rescaled forms (6.2) and (6.4) can be reconciled by equating their coefficients as follows:

When $\delta>1, A=\left(1+\delta^{2}\right) /(2 \delta)=\left(1+\rho^{2}\right) /\left|1-\rho^{2}\right|$, and $B=\left(\delta^{2}-\right.$ $1) /(2 \delta)=2 \rho /\left|1-\rho^{2}\right|$. Adding, $A+B$, and equating coefficients gives the same result as subtracting, $A-B$, namely:

$$
\delta=(1+\rho) /|1-\rho| \text { and } \rho=(\delta-1) /(\delta+1) \text { when } \delta>1 \text {; }
$$

When $\delta<1, A=\left(1+\delta^{2}\right) /(2 \delta)=\left(1+\rho^{2}\right) /\left|1-\rho^{2}\right|$, and $B=(1-$ $\left.\delta^{2}\right) /(2 \delta)=2 \rho /\left|1-\rho^{2}\right|$. Adding, $A+B$, and equating coefficients gives the same result as subtracting, $A-B$, namely:

$$
\delta=|1-\rho| /(1+\rho) \text { and } \rho=(1-\delta) /(1+\delta) \text { when } \delta<1 \text {. }
$$

To emphasize that $\delta$ and $\rho$ are functions of one another we write $\delta(\rho)$ and $\rho(\delta)$, and similarly write $\delta(1 / \rho)$ and $\rho(1 / \delta)$ to indicate the same functions evaluated at the reciprocals of their initial arguments. We find, for all $\delta \geq 0$ and all $\rho \geq 0$, that

$$
\begin{aligned}
& \text { for } \delta<1, \delta(\rho)=|1-\rho| /(1+\rho)=\delta(1 / \rho), \text { and } \\
& \text { for } \delta>1, \delta(\rho)=(1+\rho) /|1-\rho|=\delta(1 / \rho) \text { so that } \\
& \text { for any } \delta \geq 0, \delta(\rho)=\{|1-\rho| /(1+\rho)\}^{\tau}=\delta(1 / \rho) \text {, and } \\
& \text { for any } \delta \geq 0, \rho(\delta)=|1-\delta| /(1+\delta)=\rho(1 / \delta) \text {. }
\end{aligned}
$$

The unique solutions for $\delta$ and $\rho$ in terms of each other show that the classical and rescaled Cauchy distributions can comport with one another. These expressions are put in Table 1 for reference. To avoid singularities, we impose in Table 1 the restrictions

$\delta>0$ but $\delta \neq 1$, and $0<\rho<1$.

Comparing the functional expressions for the rescalers in Table 1 to the rescalers in section 3.2 shows that $\rho=r$ or $\rho=$ 
$r^{-1}$, whichever is smaller. If a calculated value for $\rho$ exceeds unity then it should be replaced with its reciprocal, as justified by $\mathrm{f}(z ; \rho, \mu, C)=\mathrm{f}\left(z ; \rho^{-1}, \mu, C\right)$, the duality shown in section 3.2 .

Table 1. Parameter Conversions for Comporting Classical ( $\mu$, $\left.\rho_{\mathrm{C}}\right)$ and Rescaled $(\mu, \delta) \operatorname{CCDs}$

\begin{tabular}{|c|c|c|}
\hline$\delta$ range & $0<\delta \neq 1$ & $0<\rho<1$ \\
\hline$\delta<1$ & $\delta=|1-\rho| /(1+\rho)$ & $\rho=(1-\delta) /(1+\delta)$ \\
\hline$\delta>1$ & $\delta=(1+\rho) / 1-\rho \mid$ & $\rho=(\delta-1) /(\delta+1)$ \\
\hline
\end{tabular}

\section{(4) Some Transforms of Classical and Rescaled CCDs}

(A). To transform $\mathrm{z} \sim \mathrm{CC}(\mu, \delta, C)$ to $\mathrm{z}^{\prime} \sim \mathrm{CC}(0,1, C) \sim$ $\mathrm{CC}(0, C)$, convert $\delta$ to the comporting precision $\rho$ using Table 1 , then put $\mu=\exp (\mathrm{i} u) \in C$ as the local complex representation of $\mu$ on $C$, and put $\zeta=\rho \mu$, so that $z \sim \mathrm{CC}(\zeta, C)$. Then by Theorem $4 z^{\prime}=T_{\mathrm{C}} z \sim \mathrm{CC}\left(T_{\mathrm{C}} \zeta, C\right)=\mathrm{CC}(0, C) \sim$ $\mathrm{CC}(0,1, C)$, because $T_{\mathrm{m}}=\left(1,-\xi^{\prime} ; \xi^{*},-1\right)$ is involutory.

(B). To transform $z \sim \mathrm{CC}(\zeta, C)$ to $z_{1} \sim \mathrm{CC}\left(\zeta_{1}, C\right)$, first transform $z$ to the uniformly distributed $z^{\prime}$ as in (A) above, then put $z_{1}=T_{\mathrm{C} 1} z^{\prime}$ with matrix $M_{\mathrm{m} 1}=\left(1,-\zeta_{1} ; \zeta_{1} *,-1\right)$. Then $z_{1} \sim \mathrm{CC}\left(\zeta_{1}, C\right)$ by theorem 4 .

(C). To transform $z \sim \mathrm{CC}\left(\mu_{1}, \delta_{1}, C\right)$ to $w \sim \mathrm{CC}\left(\mu_{2}, \delta_{2}, C\right)$, find $\zeta_{1}=\rho_{1} \mu_{1}$ conforming to $\left(\mu_{1}, \delta_{1}\right)$, and find $\zeta_{2}=\rho_{2} \mu_{2}$ conforming to $\left(\mu_{2}, \delta_{2}\right)$, as in (A). Transform $z$ to $w$ as in (B).Then $w \sim \mathrm{CC}\left(\mu_{2}, \delta_{2}, C\right)$.

(D) CCDs are closed under rotations $A S$ of $S$ : If $A^{\mathrm{T}} A=\mathrm{I}_{3}$ then $z \sim \mathrm{CC}(\mu, \delta, C) \Rightarrow A z \sim \mathrm{CC}(A \mu, \delta, A C)$, and $z \sim \mathrm{CC}(\rho \mu, C)$ $\Rightarrow A z \sim \mathrm{CC}(\rho A \mu, A C)$ since $z^{\mathrm{T}} \mu=(A z)^{\mathrm{T}}(A \mu)$ with Jacobian unity. The precision $\rho$, like the rescaler $\delta$, is not affected by rotations.

\section{SCD CONSTRUCTIONS}

\section{(1) Get Rescaled SCD from Rescaled CCD}

Definition 1: The family of rescaled SCDs is the set of probability distributions on the unit sphere $S$ that result from mappings of the form $S^{\prime}=M S$, where $S \sim S C(0,1)$, the uniform spherical distribution, and $M$ is any spherical Möbius mapping, proper or improper. We write: $S \sim \mathrm{SC}(\mu, \delta)$, where $\mu$ and $\delta$ are functions of $M$.

The set of rescaled SCDs is closed under the operation of compounding spherical Möbius functions: A SCD on a unit sphere $S_{1}=M_{1} S$, can be transformed on the left by an arbitrary spherical Möbius function $M_{2}$ to yield a SCD on the unit sphere $S_{2}, S_{2}=\mathrm{M}_{2} S_{1}$, by the group property of the spherical Möbius functions. We say that $z$ or $z_{\mathrm{S}}$ is spherical Cauchy $\mu, \delta$ on $S_{2}$, or $S_{2}$ is spherical Cauchy $\mu, \delta$, and write: $z$ or $z_{\mathrm{S}} \sim \mathrm{SC}\left(\mu, \delta, S_{2}\right)$, or $S_{2}=M S \sim \mathrm{SC}(\mu, \delta)$, where $M=M_{2} M_{1}=$ $[A, \beta, \delta]$ (basic structure), $z_{\mathrm{S}}=(\theta, \varphi), z$ and $z_{\mathrm{S}} \in S_{2}, \mu \in S_{2}, \mu$ is a function of the basic parameters of $M$ when $\delta \neq 1$, and $\mu=$ 0 (undefined) when $\delta=1$. Recall that if $\mu$ is defined the colatitude $\theta$ is by convention measured as the angular deviation of $z$ from $\mu$, forcing $\cos \theta$ to be $z^{\mathrm{T}} \mu$. The distribution on the sphere $M S$ depends, by Proposition 3 below, only on $\mu$ and $\delta$, with combined $p d f=3$, and not on the entire basic structure for $M$, whose $p d f$ is 6 .

Proposition 3: If $M$ is a spherical Möbius function then $M S \sim \mathrm{SC}(\mu, \delta)$ and is isotropically distributed about the mean axis, $\pm \mu$, where $M=[A, \beta, \delta]$ (basic structure), $\mu=M \beta=\tau A \beta$, $\tau=\operatorname{sgn}(1-\delta)$ and $S \sim \mathrm{SC}(0,1)$.

Proof: The proof is conducted in the spherical domain. If $\delta=1$ then $\tau=\mu=0, G=R_{\beta S} \Delta R_{\mathrm{S} \beta}=I_{3}, M=A$ and $S^{\prime}=A S$, a rotation of $S$ which is itself uniformly distributed, in which case the proposition is trivially true. So, assume $\delta \neq 1$.

The mapping $S^{\prime}=M S$ has four stages corresponding to the four components of the extended pseudo-polar form $M=$ $A R_{\beta \mathrm{S}} \Delta R_{\mathrm{S} \beta}$ : namely $S_{1}=R_{\mathrm{S} \beta} S, S_{2}=\Delta S_{1}, S_{3}=R_{\beta \mathrm{S}} S_{2}$, and $S^{\prime}=S_{4}$ $=A S_{3}=M S$. In stage 1 Rotor $R_{\mathrm{S} \beta}$ rotates $S$ into $S_{1}$, positioning it for rescaling in stage 2 , and rotates the base point $\beta$ on $S$ to the south pole $s$ by definition, and $-\beta$ to - s by implication. Circle-preserving properties of Proposition 1 require that $R_{\mathrm{S} \beta}$ also rotate the family of great circles through $\pm \beta$ to the family of great circles of longitude through $\pm s$. Since rotations have no effect on uniform distributions then, like $S$, $S_{1} \sim \mathrm{SC}(0,1)$.

Stage 2 is the centered rescaling about $s: S_{2}=\Delta S_{1}$, or $z^{\prime}=$ $\Delta z$, say, where $z^{\prime} \in S_{2}$ and $z \in S_{1}$. This rescaling disrupts the uniformity of the probability distributed on $S$, and in the process creates a mean axis, $\pm s$, and a mean direction $\mu$ on $S_{2}: \mu=\Delta s=\tau s=s$ if $\tau=1$ and $n$ if $\tau=-1$, according to (4) and the explanation of $\Delta s$ in section 2.5. In the planar image $\Delta_{\mathrm{E}}$ of $\Delta$ the center lines through the origin of $E$ intersect orthogonally the concentric circles about the origin; since stereographic projection is isogonal their spherical images, the great circles of longitude and the parallel circles of latitude, must also intersect at right angles on the sphere, forming a rigid grid of mutually orthogonal sets of circles as explained in section 2.2. This is true both before and after the rescaling because the rescaler $\Delta$ is a spherical Möbius mapping and therefore is itself isogonal. This process guarantees the isotropy of the distribution about the mean axis $\pm \mathrm{s}$. Then $S_{2} \sim \operatorname{SC}(\tau s, \delta)$, by (4) and by definition of the rescaled SCD.

The third stage, $S_{3}=R_{\beta S} S_{2}$, rotates the entire rescaled sphere $S_{2}$ back to its original position, now relabeled as $S_{3}$. In the process it rotates the mean axis $\pm s$ of $S_{2}$ and its rigid grid of orthogonal sets of circles to the new mean axis $\pm \beta$ and a corresponding rigid grid of orthogonal sets of circles for $S_{3}$. The rescaling is unaffected by this rotation. Since $R_{\beta S} \tau s=\beta$ when $\tau=1$, and $-\beta$ when $\tau=-1$, then $R_{\beta S} \tau s=\tau R_{\beta S} s=\tau \beta$, and $S_{3} \sim \mathrm{SC}\left(R_{\beta S} \tau s, \delta\right)=\mathrm{SC}(\tau \beta, \delta)$.

The final stage is the rotation from $S_{3}$ to $S_{4}: S_{4}=A S_{3}=$ $M S=S^{\prime} \sim \operatorname{SC}(\tau A \beta, \delta)$. The successive stages show that $\mu=$ $M \beta=\tau A \beta$, with rescaler $\delta$ and isotropy about the final mean axis $\pm \mu$. This completes the proof of Proposition 3 .

\section{(2) Probability Density Function of the Rescaled SCD}

Proposition 4: The probability density function of $z \sim$ $\mathrm{SC}\left(\mu, \delta, \mathrm{S}^{\prime}\right)$ on $S^{\prime}=M S$, where $S \sim S C(0,1)$, is

$\mathrm{f}\left(\mathrm{z} ; \mu, \delta, \mathrm{S}^{\prime}\right)=\sin \theta \times \mathrm{h}\left(\mathrm{z} ; \mu, \delta, \mathrm{S}^{\prime}\right)$, where the profile density

$\mathrm{h}\left(z ; \mu, \delta, S^{\prime}\right)=(4 \pi \kappa)^{-1}\left(\operatorname{coth} \kappa-z^{\mathrm{T}} \mu\right)^{-1}$, and 
$\delta=\exp (\kappa), z_{S}=(\theta, \varphi)$ and $\cos \theta=z^{T} \mu$.

Proof: The profile density is derived first for $\delta>1$. By Proposition $2(\mathrm{c}), \mathrm{h}\left(z ; \mu, \delta, S^{\prime}\right)$ is proportional to the variable part of the circular density (6.4) common to all the great circles of longitude $C$ on $S$ containing $\pm \mu$, and is thus proportional to $\left.\left[\left(\delta^{2}+1\right)-\left(\delta^{2}-1\right) z^{\mathrm{T}} \mu\right\}\right]^{-1}=[C+D \cos \theta]^{-1}$, say, where $C=\left(\delta^{2}+1\right), D=-\left(\delta^{2}-1\right)$ and $\delta>1$, so that

$\mathrm{h}\left(z ; n, \delta, S^{\prime}\right)=K[C+D \cos \theta]^{-1}$ where

$1 / K=\iint[C+D \cos \theta]^{-1} \sin \theta \mathrm{d} \theta \mathrm{d} \varphi$, with the integral taken over the surface of $S^{\prime}$. Simplify by substituting $w=C$ $+D \cos \theta$ for $\theta$. Then $\mathrm{d} w=-D \sin \theta \mathrm{d} \theta$, and $w$ ranges from $C+D$ to $C-D$ as $\theta$ ranges from 0 to $\pi$, and now

$-D / K=\iint w^{-1} \mathrm{~d} w \mathrm{~d} \varphi=2 \pi\{\log (C-D)-\log (C+D)\}=2 \pi$ $\log \delta^{2}$, so $K=-D /\left(2 \pi \log \delta^{2}\right)$, and

$\mathrm{h}\left(z ; n, \delta, S^{\prime}\right)=\{4 \pi \log \delta(C /(-D)-\cos \theta)\}^{-1}$,

where $C=\delta^{2}+1,-D=\delta^{2}-1$, and $\delta=\mathrm{e}^{\kappa}$. Then

$\mathrm{h}\left(z ; n, \delta, S^{\prime}\right)=\{4 \pi \kappa(\operatorname{coth} \kappa-\cos \theta)\}^{-1}, \kappa>0$,

as the profile density for $z \sim \operatorname{SC}\left(\mu, \delta, S^{\prime}\right)$ when $\delta>1$.

Next take

$\mathrm{C}=\delta^{2}+1$ as before, $\mathrm{D}=-\left(1-\delta^{2}\right)$ and $\delta<1$.

Proceeding as with the case $\delta>1, K=-\mathrm{D} /\left\{2 \pi \log \left(1 / \delta^{2}\right)\right\}$ $=\mathrm{D} /(4 \pi \kappa)$ and the profile density is exactly the same as above. This completes the proof of Proposition 4.

There is a duality in the $\operatorname{SC}(\mu, \delta, S)$ probability density that is analogous to that shown in section 3 for the circular Cauchy probability density: the $\operatorname{SC}\left(\mu, \delta, S^{\prime}\right)$ profile density function is equivalent to that for a $\operatorname{SC}\left(-\mu, \delta^{-1}, S^{\prime}\right)$ profile density function, since $\mathrm{h}\left(z^{\prime} ; \mu, \delta, S^{\prime}\right) \propto\left[\log \delta\left\{A(\delta) / B(\delta)-\mathrm{z}^{\mathrm{T}} \mu\right\}\right]^{-1}$, with $A(\delta)>B(\delta)>0$ and maximum density at $z^{\mathrm{T}} \mu=1$, while

$\mathrm{h}\left(z ;-\mu, \delta^{-1}, S^{\prime}\right) \propto\left[\log \delta^{-1}\left\{A\left(\delta^{-1}\right) / B\left(\delta^{-1}\right)-z^{\mathrm{T}}(-\mu)\right]^{-1}\right.$

$=\left[-\kappa\left\{\operatorname{coth}(-\kappa)-z^{\mathrm{T}}(-\mu)\right]^{-1}\right.$

$=\left[-\kappa\left\{-\operatorname{coth} \kappa-z^{\mathrm{T}}(-\mu)\right]^{-1}=\left[\kappa\left\{\operatorname{coth} \kappa-z^{\mathrm{T}} \mu\right\}\right]^{-1}\right.$

$\propto \mathrm{h}\left(z^{\prime} ; \mu, \delta, S\right)$, with the same proportionality constant.

To force $\kappa$ to be positive when $\delta<1$ replace $(\mu, \delta)$ with $(-\mu$, $\left.\delta^{-1}\right)$ to get $z^{\prime} \sim \operatorname{SC}\left(-\mu, \delta^{-1}, S^{\prime}\right)$, or, use $-z^{\prime} \sim \operatorname{SC}\left(\mu, \delta^{-1}, S^{\prime}\right)$, where $\delta^{-1}>1$. The parametric pairs $(\mu, \delta),\left(-\mu, \delta^{-1}\right)$ and $(-\mu,-\kappa)$ all give the same rescaled SCD. The underlying reasons for this, as explained in section 3.2, are the ambiguities in the definitions of the base and rescaler.

\section{(3) Transforms of Rescaled $\mathrm{SC}(\mu, \delta)$ Distributions}

Suppose that $M_{1} S \sim \operatorname{SC}\left(\mu_{1}, \delta_{1}\right)$ when $S \sim \operatorname{SC}(0,1)$. Given any spherical Möbius function $M_{2}$ the transform $M S=$ $M_{2} M_{1} \mathrm{~S}$ of $M_{1} S$ will be $\mathrm{SC}(\mu, \delta)$ for some $(\mu, \delta)$. We seek to determine the parameters $(\mu, \delta)$ for $M S$ when $M_{2}$ is given and $\left(\mu_{1}, \delta_{1}\right)$ are known or estimable, but the entire basic structure of $M_{1}$ is neither known nor estimable. A stand-in Möbius spherical function for $M_{1}$ will be designed to solve this problem, It will be denoted by $\left[\mu_{1}, \delta_{1}\right]$ and will be called a mimetic mapping for $M_{1}$ or $\left(\mu_{1}, \delta_{1}\right)$, or simply a mimetic for short. Its parameters must be strictly limited to $\left(\mu_{1}, \delta_{1}\right)$ since any additional parameters would confound the results. Similarly, $\left[\mu_{1}, \delta_{1}\right] S$ and $M_{2}\left[\mu_{1}, \delta_{1}\right] S$ are mimetics for $M_{1} S$ and $M_{2} M_{1} S$. The mimetic $\left[\mu_{1}, \delta_{1}\right]$ for $M_{1}$ must satisfy (a) $\left[\mu_{1}, \delta_{1}\right] S \sim M_{1} S \sim \operatorname{SC}\left(\mu_{1}, \delta_{1}\right)$, and

(b) $M_{2}\left[\mu_{1}, \delta_{1}\right] S \sim M_{2} M_{1} S \sim \operatorname{SC}(\mu, \delta)$ for some $(\mu, \delta)$, and

for any given $M_{2}$ and for every $M_{1}$ for which $M_{1} S \sim$ $\operatorname{SC}\left(\mu_{1}, \delta_{1}\right)$. Actually, it suffices if just (a) above is shown to be satisfied by $\left[\mu_{1} . \delta_{1}\right]$, since then the mimetic $\left[\mu_{1}, \delta_{1}\right] S$ for $M_{1} S$ and $M_{1} S$ itself provide identical inputs to $M_{2}$, so the outputs in (b) will be the same. The parameters for the mimetic are restricted to $\mu_{1}$ and $\delta_{1}$ to guarantee that (a) $\Rightarrow(b)$. Indeed, (b) $\Rightarrow$ (a) also since (b) must hold for any $M_{2}$, and we can choose $M_{2}$ to be the identity mapping.

The existence of a mimetic $\left[\mu_{1}, \delta_{1}\right]$ satisfying (a) is crucial, otherwise no transformations can be done and the rescaling properties for the SCD will be of little use. Fortunately such a mimetic does exist.

Proposition 5: Let $M_{1}$ be any spherical Möbius function with $\mathrm{S}_{1}=\mathrm{M}_{1} \mathrm{~S} \sim \mathrm{SC}\left(\mu_{1}, \delta_{1}\right)$ where $\left(\mu_{1}, \delta_{1}\right)$ are known or estimable. If $\delta_{1}=1$ then $[0,1]=I_{3}$ is a positive hermitian mimetic for $M_{1}$; If $\delta_{1} \neq 1$ then $\left[\mu_{1}, \delta_{1}\right]=R_{\mu 1 S} \Delta_{1}^{\prime} R_{\mathrm{S} \mu 1}$ and $\left[\mu_{1}, \delta_{1}\right]^{-1}$ are positive hermitian mimetics for $M_{1}$ and $M_{1}^{-1}$, where $\Delta_{1}^{\prime} \mathrm{s}$ $\equiv \mathrm{s}$ (no $\tau$ involved). These positive hermitian mimetics are unique.

Proof: If $\delta_{1}=1$ then the "parameters" for $\left[\mu_{1}, \delta_{1}\right]$ can only be the constants 0 and 1 . Any $3 \times 3$ aligner matrix $A$ is a mimetic since $A S \sim \mathrm{SC}(0,1)$. But the mimetic $[0,1]=\mathrm{I}_{3}$ is the only positive hermitian aligner whose parameters are 0 and 1 , and $\mathrm{I}_{3} S \sim \mathrm{SC}(0,1)$ as required by (a).

If $\delta_{1} \neq 1$ a subtle quirk appears: since $\mu_{1}$ is known its algebraic sign is fixed and must not be changed by $\tau$ in the definition of $\mu$ in (4), thus prohibiting the use of $\tau$ in the mimetic for $M_{1}$ but requiring its use in $M_{1}$. Then $\Delta_{1} s=\tau s$ for $M_{1}$, but, as a definition of $\Delta_{1}^{\prime}$, we use $\Delta_{1}^{\prime} s=s$ for the mimetic $\left[\mu_{1}, \delta\right]$. Suppose $M_{1}=A_{1} R_{\beta 1 \mathrm{~S}} \Delta_{1} R_{\mathrm{S} \beta 1}$ (extended pseudo-polar form), so that $M_{1} \beta_{1}=\mu_{1}$ with rescaler $\delta_{1}$. The base $\beta_{1}^{\prime}$, say, for the mimetic must be either $s$ or $\mu_{1}$ since $\beta_{1}$ is unknown. If $\beta_{1}^{\prime}=s$ then the rotor for the mimetic must be the identity matrix since it maps $s$ to $s$, and the extended pseudo-polar form for the mimetic $\left[\mu_{1}, \delta_{1}\right]$ is $R_{\mu 1 S} \mathrm{I}_{3} \Delta_{1}^{\prime} \mathrm{I}_{3}$, which is not positive hermitian. The only alternative is to use $\beta_{1}^{\prime}=\mu_{1}$ for the mimetic base. This forces the unique positive hermitian mimetic to be $\left[\mu_{1} . \delta_{1}\right]=R_{\mu 1 S} \Delta^{\prime}{ }_{1} R_{\mathrm{S} \mu 1}$. Since (b) $\Rightarrow$ (a) then, on setting $M_{2}=\left[\mu_{1}, \delta_{1}\right]^{-1}$ in (b) we have $S=\left[\mu_{1}, \delta_{1}\right]^{-1}\left[\mu_{1}, \delta_{1}\right] S \sim$ $\left[\mu_{1}, \delta_{1}\right]^{-1} M_{1} S$. This completes the proof of Proposition 5 .

Some examples, where it is assumed that $S \sim \operatorname{SC}(0,1)$ :

(A) To convert $M_{1} S \sim \mathrm{SC}\left(\mu_{1}, \delta_{1}\right)$ to $\mathrm{SC}(0,1)$, a uniform distribution: By Proposition 5, $\left[\mu_{1}, \delta_{1}\right]^{-1} M_{1} S \sim S$.

(B) To transform the sphere $M_{1} S \sim \operatorname{SC}\left(\mu_{1}, \delta_{1}\right)$ to a $\operatorname{SC}\left(\mu_{2}, \delta_{2}\right)$ distribution, put $\left[\mu_{1}, \delta_{1}\right]=R_{\mu 1 S} \Delta_{1}^{\prime} R_{\mathrm{S} \mu 1}$ as in (A) above, and put $\left[\mu_{2}, \delta_{2}\right]=\left[I_{3}, \mu_{2}, \delta_{2}\right]$ (basic structure) $=$ $R_{\mu 2 S} \Delta_{2}^{\prime} R_{\mathrm{S} \mu 2}$. Then $\left[\mu_{1}, \delta_{1}\right]^{-1} M_{1} S \sim \mathrm{S} \sim \mathrm{SC}(0,1)$ by $(\mathrm{A})$, and $\left[\mu_{2}, \delta_{2}\right]\left\{\left[\mu_{1}, \delta_{1}\right]^{-1} M_{1} S \sim \operatorname{SC}\left(\mu_{2}, \delta_{2}\right)\right.$ by Proposition 5 .

(C) To get the parameters $(\mu, \delta)$ for the distribution of $M S$ where $M S=M_{2} M_{1} S \sim \operatorname{SC}(\mu, \delta)$, given $M_{2}$ and $\left(\mu_{1}, \delta_{1}\right)$. First compute $M_{2}\left[\mu_{1}, \delta_{1}\right]$ where $\left[\mu_{1}, \delta_{1}\right]=R_{\mu 1 S} \Delta^{\prime}{ }_{1} R_{\mathrm{S} \mu 1}$ as in (A) and (B). Then $M_{2}\left[\mu_{1}, \delta_{1}\right] S \sim M S \sim \mathrm{SC}(\mu, \delta)$ by (b) above. To get $(\mu, \delta)$ we must get the basic structure of the Möbius matrix $M_{\mathrm{m}}$ for the planar image $M_{\mathrm{E}}$, say, where $M_{2}\left[\mu_{1}, \delta_{1}\right] \leftrightarrow M_{\mathrm{E}}$. Put $M_{\mathrm{m}}=\left[A_{\mathrm{m}}, \beta_{\mathrm{E}}, \delta\right]$ (basic structure). See the Appendices for how to calculate the components of this basic structure. The rotor 
$R_{\mathrm{m} 0 \beta}$ is obtained from $\beta_{\mathrm{E}}$ as per the example following theorem 3 in section 2.6. The spherical rotation images, $A$ and $R_{\mathrm{S} \beta}$, of $A_{\mathrm{m}}$ and $R_{\mathrm{m} 0 \beta}$, are obtained using the formulae of Appendices II(A) and II(E). The mean direction is $\mu=\tau A \beta$, where $\tau$ is obtained from $\delta$, and $\beta$ from $\beta_{\mathrm{E}}$ using the equations in section 2.2.

\section{(4) Some Properties of the $\operatorname{SC}(\mu, \delta)$ Distribution}

The orthogonal angles $\theta$ and $\varphi$ are independent, and $\varphi$ is uniformly distributed over a unit circle for all rescaled SCDs. The expected value of $z$ when $z \sim \operatorname{SC}(\mu, \delta, S)$ is

$E(z ; \mu, \delta, S)=\rho_{\mathrm{S}} \mu$, where $\delta=\mathrm{e}^{\kappa}$ and $\rho_{\mathrm{S}}=\operatorname{coth} \kappa-1 / \kappa$

This unexpected result shows that the precision $\rho_{\mathrm{S}}$ for the SCD has the same form as that for the spherical von-Mises Fisher distribution. Equation (9) can be shown directly by integration of the probability density for $z$ in Proposition 4, using the same substitution, $w=C+D \cos \theta$, as before.

If $S \sim \mathrm{SC}(\mu, \delta)$ then all the great circles on $S$ passing through $\pm \mu$ will be $\mathrm{CC}(\mu, \delta)$. Analogous relationships hold for the great circles through $\pm \mu$ of a sphere with the von Mises-Fisher spherical probability distribution (see [11] for details), whose profile density is proportional to $\exp \left(\kappa z^{\mathrm{T}} \mu\right)$. Another similarity between the two families is that, for the great circles and spheres through $\mu$ of both families, the ratio of the maximum profile density at $z=\mu$ to the minimum at $z$ $=-\mu$ is always $\exp (2 \kappa)$, or $\delta^{2}$. Despite these similarities the spherical and circular Cauchy densities have taller and narrower peaks and contain more tail probability than their corresponding von-Mises Fisher distributions.

The same parametric notation, $(\mu, \delta)$, is used for both the $\operatorname{CC}(\mu, \delta)$ and $\operatorname{SC}(\mu, \delta)$ distributions, even though they have the same rescaler but different precisions. Knowledge of any one of the three parameters $\rho, \rho_{\mathrm{S}}$ or $\delta$ is sufficient to determine the other two. This is so because the spherical precision $\rho_{\mathrm{S}}$ and the circular precision $\rho$ can be expressed in terms of their common rescaler $\delta$ (or concentration parameter $\kappa=\log$ $\delta$ ), which is tantamount to indirectly expressing them in terms of each other. Since Table 1 implies that $\rho=|\delta-1| /(\delta$ +1 ), we can infer that

$\rho=\tanh |\kappa| / 2$, while $\rho_{\mathrm{S}}=\operatorname{coth} \kappa-1 / \kappa$ and $\delta=\mathrm{e}^{\kappa}$.

To show the first of equations (10), assume $\delta>1$, so $\kappa>$ 0 . Then $\rho=(\delta-1) /(\delta+1)$. Substitute $\mathrm{e}^{\mathrm{\kappa}}$ for $\delta$; and then multiply numerator and denominator by $\mathrm{e}^{-\kappa / 2}$ to get $\rho=\tanh \kappa / 2$ $=\tanh |\kappa| / 2$. If $\delta<1$ then $\rho=(1-\delta) /(1+\delta)$ and $\kappa<0$. Substitute $\mathrm{e}^{-|\kappa|}$ for $\delta$, and multiply thoughout by $\mathrm{e}^{|\kappa| / 2}$ to get the same result, $\rho=\tanh |\kappa| / 2$. Equations (10) hold for all $\delta>0$ except $\delta=1$, for all $\kappa \in(-\infty, \infty\}$ except $\kappa=0$ and for all $\rho \in(0,1)$. If $\kappa$ is given then $\rho$ and $\rho_{\mathrm{S}}$ can be calculated directly from (10). Given $\rho, \kappa$ can be found via the inverse function

$|\kappa|=2 \operatorname{atanh} \rho=\log \{(1+\rho) /(1-\rho)\}$,

and $\rho_{S}$ then calculated from (10). Given $\rho_{S},|\kappa|$ can be determined from (10) by iteration, and $\rho$ then calculated directly from (10). The equations in (10) hold even if the mean directions for the circular and spherical distributions are not the same.

\section{(5) Special Circle and Sphere Projection Mappings}

The classical SCD is defined in section 4.7 below via the special Möbius circle mapping $T_{\mathrm{C}}$. The involutory matrix of this mapping is $T_{\mathrm{m}}=\left(1,-\zeta ; \zeta^{*},-1\right)$, where $\zeta=r \exp (\mathrm{i} u), r \neq$ 1 and $u \in(-\pi, \pi]$. This mapping was briefly described in theorem 4 of section 2.8, and it will hereafter be called a (circle) $\zeta$-projection, with projection point $\zeta$, a name motivated by the well-known defining property of $T_{\mathrm{C}}$ manifested in

Theorem 5: The Möbius mapping $\mathrm{z}^{\prime}=T_{\mathrm{C}} \mathrm{z}$ is a $\zeta$ projection $\Leftrightarrow T_{\mathrm{C}}$ maps a unit circle $C$ one-one and onto itself (relabeled as $C^{\prime}, C^{\prime}=T_{\mathrm{C}} C$ ) by means of the straight line projection making the three points $\left(z^{\prime}, z, \zeta\right)$ collinear in the plane $F$ whose unit circle is $C$, with projection point $\zeta$ any complex point in $F$ that is not on $C$.

Proof: $(\Rightarrow)$ Assume $T_{\mathrm{C}}$ is a $\zeta$-projection, so its matrix is $\left(1,-\zeta ; \zeta^{*},-1\right)$, and $\left|T_{\mathrm{m}}\right| \neq 0$. For any $z$ on $C$,

$$
\begin{aligned}
& z^{\prime}=(z-\zeta) /\left(\zeta^{*} z-1\right)=(z-\zeta) /\left\{z\left(\zeta^{*}-z^{*}\right)\right\} \Leftrightarrow \\
& z^{\prime} z=-(z-\zeta) /\left(z^{*}-\zeta^{*}\right)=-(z-\zeta)^{2} /\left|z^{*}-\zeta *\right|^{2} \Leftrightarrow \\
& \left(z^{\prime} z\right)^{1 / 2}=\mathrm{i} Z \text {, say, where } Z=(z-\zeta) /|z-\zeta| \text { and }|Z|=1 .
\end{aligned}
$$

Then $\left\{\left|z^{\prime}\right|=1\right.$ and $\left.z^{\prime} \in C^{\prime}\right\} \Leftrightarrow\left\{T_{\mathrm{C}}\right.$ is a circle mapping from $C$ to itself, $\left.C^{\prime}\right\}$. Further,

$$
\begin{aligned}
& {\left[\left\{\left(z^{\prime} z\right)^{1 / 2}\|\mathrm{i} Z \perp Z\| z-\zeta\right\} \Leftrightarrow\left\{\left(z^{\prime} z\right)^{1 / 2} \perp(z-\zeta)\right\}\right. \text { and }} \\
& \left.\left\{\left(z^{\prime} z\right)^{1 / 2} \| z^{\prime}+\mathrm{z} \perp z^{\prime}-z\right\} \Leftrightarrow\left\{\left(z^{\prime} z\right)^{1 / 2} \perp z^{\prime}-z\right\}\right] \Leftrightarrow \\
& z-\zeta \| z^{\prime}-z,
\end{aligned}
$$

the last line presenting two parallel line segments with a common point. This occurs if and only if the three points $\left(z^{\prime}\right.$, $z, \xi)$ involved are collinear. The one-one, onto and circle preserving Möbius Properties apply to all Möbius functions, proper or improper, and thus to $T_{\mathrm{C}}$ by theorem 1 .

$(\Leftarrow)$ The collinearity of $\left(z^{\prime}, z, \zeta\right)$ emulates the functional $T_{\mathrm{C}}$ mapping of $C$ to itself because to every point $z \in C$ the collinearity assigns a unique point $z^{\prime} \in C^{\prime}$. And, the relevant implication symbols in the proof above are all two-way.

Now define a spherical $\zeta$-projection mapping $T$ analogous to the defining property of the circular $\zeta$-projection $T_{\mathrm{C}}$ of theorem 5 by:

Definition 2: A spherical mapping $T$ is a $\zeta$-projection, $z^{\prime}$ $=T z$, if it maps a unit sphere $S$ onto itself (relabeled as $S^{\prime}, S^{\prime}$ $=T S$ ) by means of the straight line projection making the three points $\left(z^{\prime}, z, \zeta\right)$ collinear in $x y$ t-space, with projection point $\zeta=r \mu$ any point along the positive $\mu$ axis that is not $\mu$.

The same notation, $\zeta$, for the projection point has been deliberately used here to represent both the complex point $\zeta$ $=r \exp (\mathrm{i} u)$ for classical CCDs and the real 3-vector $\zeta=r \mu$ for classical SCDs. The vector $r \mu$ and the complex point $r$ $\exp (\mathrm{iu})$ represent the same point expressed in different coordinate systems. When $\zeta$ is considered as complex it has 2 $p d f$, and when considered as a point in $x y t$-space it has $3 p d f$. When both circular and spherical $\zeta$-projection points are involved we either distinguish them or we use the interpretation, complex number or real vector, that is most appropriate and convenient.

\section{(6) Geometry of Circular and Spherical $\zeta$-projections}

Fig. (2) shows the key points on the coincident $C$ and $C^{\prime}$ circles for the special circle mapping $C^{\prime}=T_{\mathrm{C}} C$. The $\pm \mu$ axis is the perpendicular bisector of the chord of $C^{\prime}$ joining the tangent points $z_{\mathrm{T} 1}$ and $z_{\mathrm{T} 2}$. The projection point $\zeta$ is the center 
of gravity $\rho \mu$ inside $C$, or is $\rho^{-1} \mu$ outside $C$. The points $\rho \mu$ and $\rho^{-1} \mu$ are reflections of one another in $C^{\prime}$ since they both lie on the $+\mu$ ray, and the product of their labeled lengths, $\rho^{-1}$ and $\rho$, is indeed equal to unity by the similarity of the right triangles whose vertices are $\left(0, \rho^{-1} \mu, \mathrm{z}_{\mathrm{T} 1}\right)$ and $\left(0, \rho \mu, z_{\mathrm{T} 1}\right)$. The duality of the $\mathrm{CC}(\zeta)$ distribution means, in the present context, that either $\rho \mu$ or $\rho^{-1} \mu$ can be used as the legitimate projection point $\xi$.

$\operatorname{Arc}\left(z_{\mathrm{T} 1}, \mu, z_{\mathrm{T} 2}\right)$ is the cap of circle $C^{\prime}, \operatorname{arc}\left(\mathrm{z}_{\mathrm{T} 1},-\mu, z_{\mathrm{T} 2}\right)$ is the cup, and $C^{\prime}$ the union of the cap and cup. Points $z$ on the cap are mapped by $T_{\mathrm{C}}$ one-one to points $z^{\prime}$ on the cup, by the collinearity of the three points $\left(z^{\prime}, z, \zeta\right)$, and conversely, regardless of which point, $\rho \mu$ or $\rho \mu^{-1}$, is the projection point $\xi$.

Applying $T_{\mathrm{C}}$ to $C^{\prime}$ maps the points back to their original positions, illustrating that $T_{\mathrm{C}}$ is involutory. As $\rho$ decreases the cap gets relatively larger and becomes equal to the cup when $\rho$ is zero - a uniform distribution. The probability density is a minimum at $z^{\prime}=-\mu$ and from there increases monotonically and symmetrically about the $\pm \mu$ axis to a maximum at $z^{\prime}=\mu$.

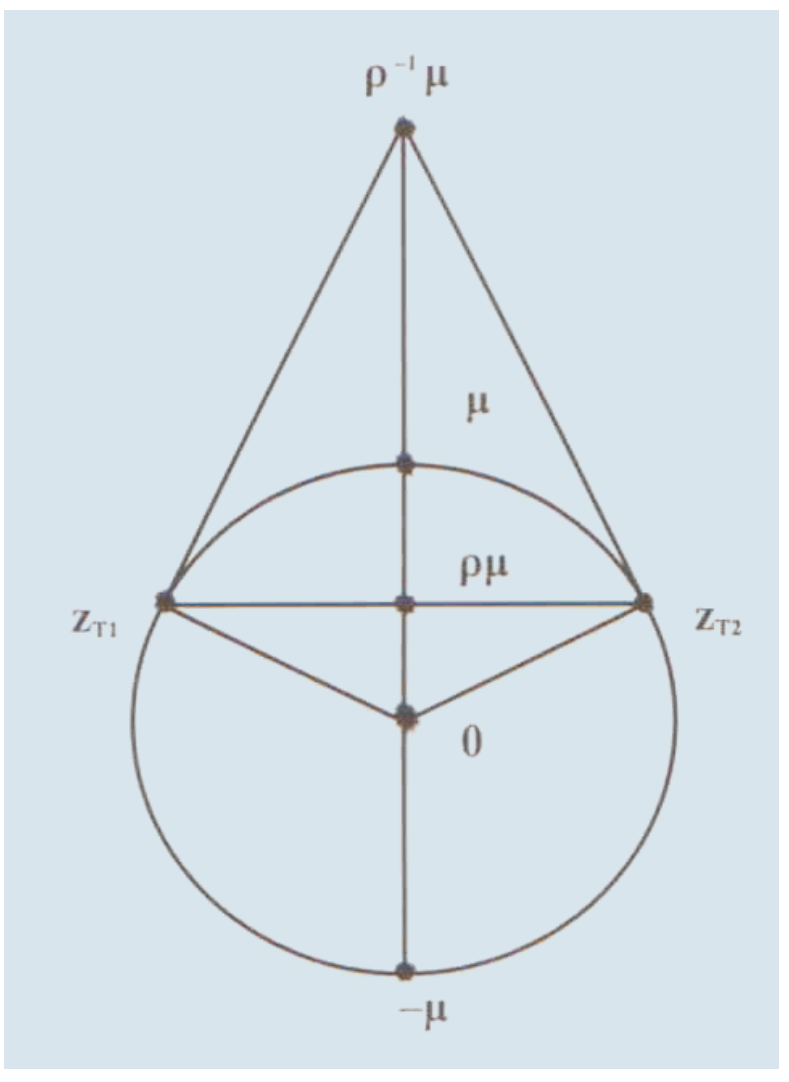

Fig. (2). Geormetry of Classical Directional Cauchy Distributions.

Circular $\zeta$-projection properties change markedly with the position of the projection point $\zeta$, which is inside, on or outside $C$. $\mu$.

If $r=1, \zeta$ is on $\mathrm{C}$, the mapping is degenerative, and $z^{\prime} \equiv$

When $r<1$ the projection point $\zeta=\rho \mu$ and is inside $C^{\prime}$. As the input point $z$ moves CCW from $\mu$ to $-\mu$ to $\mu$ on $C$ the output z' also moves CCW on $C^{\prime}$, from $-\mu$ to $\mu$ to $-\mu$, and $\zeta$ is always between $z$ and $z^{\prime}$. This Möbius mapping is proper. If
$C \sim \mathrm{CC}(0)$ the cap has probability $\approx 1 / 3$, and the cup $\approx 2 / 3$. For $C^{\prime}$ the probabilities are reversed: cap $\approx 2 / 3$ and cup, $1 / 3$.

When $r>1$ the projection point $\zeta=\rho^{-1} \mu$ and is outside $C^{\prime}$. As the input point $z$ on $C$ moves CCW from $z_{\mathrm{T} 2}$ to $z_{\mathrm{T} 1}$ and continues CCW around $C$ and back to $z_{\mathrm{T} 2}$, the output point $z^{\prime}$ of $C^{\prime}$ moves $\mathrm{CW}$ from $z_{\mathrm{T} 2}$ to $z_{\mathrm{T} 1}$ and continues $\mathrm{CW}$ back to $z_{\mathrm{T} 2}$, all the while maintaining collinearity of the three points $\left(z^{\prime}, z, \zeta\right)$. This mapping is therefore improper. Since probability is uniformly distributed over $C$ the cap still has a probability of about $1 / 3$ and the cup, about $2 / 3$. The probabilities are still reversed for $C^{\prime}$, where the cap has a probability of about $2 / 3$, and the cup $1 / 3$. This improper mapping has $z_{\mathrm{T} 1}$ and $z_{\mathrm{T} 2}$ as its fixed points.

To conceptualize the spherical $\zeta$-projection in terms of circular $\zeta$-projections, let $\mu$ be any fixed point on the surface of the sphere $S$, and consider the family of unit circles $C$ on $S$ which pass through the antipodal points $\pm \mu$. Each such $C$ has associated with it an extended complex plane $F$ whose unit circle is $C$. Let $\zeta=r \mu$ be any point of xyt-space except $\mu$ along the positive ray of the $\pm \mu$ axis. The points $(-\mu, \mu, \zeta)$ are collinear, and the extended line through them is common to every extended complex plane $F$. The union of the $C$ circles is $S$, and their intersection is $\pm \mu$; the union of the complex $F$ planes is all of xyt-space, and their intersection is the extended line through $(-\mu, \mu, \zeta)$. The cap and cup circular arcs become a spherical cap and spherical cup, and the sphere their union. The endpoints $\mathrm{z}_{\mathrm{T} 1}$ and $\mathrm{z}_{\mathrm{T} 2}$ of the chord become a parallel circle of latitude, and the union of the tangent lines $\left(\rho^{-1} \mu\right.$ to $\left.z_{\mathrm{T} 1}\right)$ and $\left(\rho^{-1} \mu\right.$ to $\left.z_{\mathrm{T} 2}\right)$ is a circular cone. The circles $C$ through the mean axis $\pm \mu$ now correspond, by convention, to the great circles of longitude on $S$.

\section{(7) Get Rescaled SCD from Classical SCD}

The infrastructure of the spherical $T$ and circular $T_{\mathrm{C}} \zeta$ projections and the duality of the projection point $\zeta$ notation become clearer with this

Lemma: Let $\zeta=r \mu, r \neq 1$. A spherical mapping $T, S^{\prime}=T S$, $\mathrm{S} \sim \mathrm{SC}(0)$, is a spherical $\zeta$-projection $\Leftrightarrow T$ induces identical circular $\xi$-projections $T_{\mathrm{C}}$ on each great circle of longitude $C$ $\left(C^{\prime}=T_{\mathrm{C}} C\right)$ that passes through $\pm \mu$ on $S$.

Proof $(\Rightarrow)$ Assume that $T$ is a $\zeta$-projection. This means that for every $z \in S, z^{\prime}=T z \in S^{\prime}$, and $\left(z^{\prime}, z, \zeta\right)$ are collinear for a projection point vector $\xi$. We must show this implies that, for any $z_{\mathrm{C}}$ on any circle $C$ through $\pm \mu$ there exists a point $z_{\mathrm{C}}^{\prime}$ $\in C$ for which $\left(z_{C}^{\prime}, z_{C}, \zeta_{\mathrm{E}}\right)$ are collinear for a complex projection point $\zeta_{\mathrm{E}}$ that is the same point as $\zeta$.

Let $C$ be any circle through $\pm \mu$ and $z_{C}$ any point on $C$. If $z_{\mathrm{C}}$ is $\mu$ or $-\mu$ then $z_{\mathrm{C}}^{\prime}$ is $-\mu$ or $\mu$ and $\left(-\mu, \mu, \zeta_{\mathrm{E}}\right)$ are automatically collinear. If $z_{\mathrm{C}}$ is a point of tangency for the line joining $z_{\mathrm{C}}$ to $\zeta_{\mathrm{E}}$ then $z_{\mathrm{C}}^{\prime}=z_{\mathrm{C}}$ and again $\left(z_{\mathrm{C}}^{\prime}, z_{\mathrm{C}}, \zeta_{\mathrm{E}}\right)$ are collinear. Assume then that the point $z_{C}$ is neither a tangent point to $C$ nor $\pm \mu$. Then $z_{\mathrm{C}} \in S$ since $C \subset S$. And $\left\{z_{\mathrm{C}}, \zeta_{\mathrm{E}}\right\} \in F$, where $F$ is the extended complex plane whose unit circle is $C$, because $C \subset F, \zeta_{\mathrm{E}}$ is on the line through $\pm \mu$, and this line is common to every extended complex plane whose unit circle contains $\pm \mu$. Since $\left\{z_{\mathrm{C}}, \zeta_{\mathrm{E}}\right\} \in F$ there must, by hypothesis, be a unique point $z^{\prime} \in S^{\prime}$ for which $\left(\mathrm{z}^{\prime}, z_{\mathrm{C}}, \zeta_{\mathrm{E}}\right)$ are collinear. This implies $z^{\prime}$ $\in F$. Since $z^{\prime} \in \mathrm{S}^{\prime}$ then $\left|z^{\prime}\right|=1$, and therefore $z^{\prime}$ must be on $C$, 
and must be $z^{\prime}$, the unique point $z^{\prime}{ }_{C} \in C$ for which $\left(z^{\prime}{ }_{C}, z_{C}, \zeta_{\mathrm{E}}\right)$ are collinear for $z_{\mathrm{C}}$ on $C$.

$(\Leftarrow)$ : Assume that $T_{\mathrm{C}}$ is a $\zeta$-projection. For any great circle of longitude $C$ through $\pm \mu$, and any point $z$ on $C$, we have that $z^{\prime}=T_{\mathrm{C}} z$ and $\left(z^{\prime}, z, \xi\right)$ are collinear for complex $\zeta$. We must show this collinearity holds also on the sphere $S$ for a vector $\zeta$. Since $T_{\mathrm{C}}$ is a $\zeta$-projection then both $z, z^{\prime} \in C \subset S$. And $\zeta$ is in every $F$. Thus the points $\left(z^{\prime}, z, \zeta\right)$ are collinear on $S$ for a vector $\zeta$.

Definition 3: The classical spherical Cauchy distributions (classical SCDs) are defined as the set of probability distributions on the unit sphere $S^{\prime}$ that are induced by spherical $\zeta$-projections of the form $S^{\prime}=T S$ from $S$ to itself (relabeled as $S^{\prime}$ ), where $S \sim \mathrm{SC}(0,1)$. We say that $S^{\prime}$, or $z^{\prime}$ or $z_{S}^{\prime}$ is classical spherical Cauchy $\zeta$, and write: $S^{\prime} \sim \operatorname{SC}(\zeta)$, or $\mathrm{z}^{\prime}$ or $z_{\mathrm{S}}^{\prime} \sim \mathrm{SC}\left(\zeta, S^{\prime}\right)$.

The projection point $\zeta$ may be either complex or vectorvalued, whichever is most appropriate and convenient. Both classical SCDs and CCDs are degenerate when the projection point $\zeta$ lies on $S$, are proper when $\zeta$ is inside $S$, and improper when $\zeta$ is outside $S$. The parameters for the classical CCDs and SCDs are the same: $(\mu, \rho)$ or $(\mu, r)$, as are the parameters for the rescaled CCDs and SCDs: $(\mu, \delta)$.

Proposition 6: The classical $\mathrm{SC}\left(\zeta, S^{\prime}\right)$ distribution is identical to the rescaled $\mathrm{SC}\left(\mu, \delta, S^{\prime}\right)$ distribution when $\zeta=r \mu$ and the parameters $\rho$ and $\delta$ comport as in Table 1 , in which case $\zeta=\rho \mu$ or $\zeta=\rho^{-1} \mu$ and $\rho=\min \left(r, r^{-1}\right), r \neq 1$.

Proof: The profile density induced on $S^{\prime}$ by $T$ is proportional to a $\mathrm{CC}(\zeta)$ density (put $\zeta_{1}=0$ in theorem 4). This $\mathrm{CC}(\zeta)$ profile density is proportional to the comporting $\mathrm{CC}(\mu, \delta)$ density in section 3.3 , which in turn is proportional to the rotated $\mathrm{CC}(\mu, \delta)$ density of Proposition 2(c). This latter density was used to derive the rescaled probability density function for a $\operatorname{SC}\left(\mu, \delta, S^{\prime}\right)$ distribution in Proposition 4, which proves Proposition 6.

\section{(8) Transformations of Classical SCDs}

The classical and rescaled SCDs have different forms, but nevertheless are identical and comport with one another by Proposition 6. The dualities of the two forms are also different, but change appropriately when either form is converted to the other. The classical CCDs and SCDs can be converted to and fro between proper and improper at the discretion of the investigator by swapping the projection points $\rho \mu$ and $\rho^{-1} \mu$. Proper rescaled CCDs and SCDs can be made improper by the reflection $z^{\prime}=J z$.

Mimetics are available for dealing with rescaled SCDs, and the mimetics themselves are spherical Möibus mappings. It would be difficult to find replacements for these mimetics with such desirable properties, so mimetics will be used to conduct transformations of classical and rescaled SCDs.

Some examples of transformations of SCDs:

(A). Transform a rescaled SCD, $z_{1} \sim \mathrm{SC}\left(\mu_{1}, \delta_{1}, S\right)$, to the uniform distribution, $\mathrm{Z}_{1}^{\prime} \sim \mathrm{SC}\left(0,1, S^{\prime}\right) \sim \mathrm{SC}\left(0, S^{\prime}\right)$. This was done with mimetics in section 4.3. This task can be dealt with using the involutoric $\zeta$-projection $T$. Convert $\delta_{1}$ to the comporting precision $\rho_{1}$ using Table 1 , and put $\zeta_{1}=\rho_{1} \mu_{1}$. Let $T_{1}$ be the $\zeta_{1}$-projection. Express the distribution of $z_{1}$ in the equivalent classical form: $z_{1} \sim \operatorname{SC}\left(\zeta_{1}, S\right)$. Then $z_{1}^{\prime}=T_{1} z_{1} \sim$ $\mathrm{SC}\left(T_{1} \zeta_{1}, S^{\prime}\right)=\mathrm{SC}\left(T_{1} T_{1} 0, S^{\prime}\right)=\mathrm{SC}\left(0, S^{\prime}\right) \sim \mathrm{SC}\left(0,1, S^{\prime}\right)$ since spherical $\zeta$-projections, like circular $\zeta$-projections, are involutory.

(B). Transform a given distribution $z_{1} \sim \operatorname{SC}\left(\zeta_{1}, S_{1}\right)$ to a prescribed distribution $z_{2} \sim \operatorname{SC}\left(\zeta_{2}, S_{2}\right)$. Put $\zeta_{1}=\rho_{1} \mu_{1}$ where $\rho_{1}$ comports with $\delta_{1}$, and put $\zeta_{2}=\rho_{2} \mu_{2}$ where $\rho_{2}$ comports with $\delta_{2}$. Express the conversions of $\mathrm{z}_{1}$ and $\mathrm{z}_{2}$ from classical to rescaled as: $\mathrm{z}_{1} \sim \mathrm{SC}\left(\mu_{1}, \delta_{1}, S_{1}\right)$ and $\mathrm{z}_{2} \sim \mathrm{SC}\left(\mu_{2}, \delta_{2}, S_{2}\right)$. Put $\Delta_{2}^{\prime}=$ $\operatorname{Diag}\left(\delta_{2}, 1\right)$ and $\Delta_{1}^{\prime}{ }^{-1}=\operatorname{Diag}\left(\delta_{1}{ }^{-1}, 1\right)$, where the primes indicate that $\tau$ is not to be used in the rescalings. Then

$$
S_{2}=R_{\mu 2 \mathrm{~S}} \Delta_{2}^{\prime} \Delta_{1}^{\prime}{ }_{1}^{-1} R_{\mathrm{S} \mu 1} S_{1} \sim \mathrm{SC}\left(\mu_{2}, \delta_{2}\right) \sim \mathrm{SC}\left(\zeta_{2}\right) .
$$

The steps in this succession of spherical operators will be examined in detail. First we note by theorem 3 and the definition of the rescaled SCD that $S_{1}=\left(R_{\mu 1 \mathrm{~S}} \Delta_{1}^{\prime} \mathrm{R}_{\mathrm{S} \mu 1}\right) S \sim$ $\operatorname{SC}\left(\mu_{1}, \delta_{1}\right)$, when $S \sim \operatorname{SC}(0,1)$, and thus

$$
\begin{aligned}
& S_{2}=\left(R_{\mu 2 \mathrm{~S}} \Delta_{2}^{\prime} \Delta_{1}^{\prime}{ }_{1}^{-} 1 R_{\mathrm{S} \mu 1}\right) S_{1} \text {; that is, in succession: } \\
& R_{\mathrm{S} \mu 1} S_{1} \sim \operatorname{SC}\left(s, \delta_{1}\right), \\
& \Delta_{1}^{\prime}{ }_{1}^{-1} R_{\mathrm{S} \mu 1} S_{1} \sim \mathrm{SC}(0,1), \\
& \Delta_{2}^{\prime} \Delta_{1}^{\prime}{ }_{1}^{-1} R_{\mathrm{S} \mu 1} S_{1} \sim \mathrm{SC}\left(s, \delta_{2}\right), \\
& R_{\mu 2 \mathrm{~S}} \Delta_{2}^{\prime} \Delta_{1}^{\prime}{ }_{1}^{-1} R_{\mathrm{S} \mu 1} S_{1}=S_{2} \sim \operatorname{SC}\left(\mu_{2}, \delta_{2}\right) .
\end{aligned}
$$

(C). Compound the $\zeta$-projections

$T_{1}$, a $\zeta_{1}$-projection of $S$ onto $S_{1}$, and

$T_{2}$, a $\zeta_{2}$-projection of $S_{1}$ onto $S_{2}$, into the single projection

$T$, a $\zeta$ projection of $S$ onto $S_{2}$.

Put $\zeta_{1}=\rho_{1} \mu_{1}$ where $\rho_{1}$ comports with $\delta_{1}, \zeta_{2}=\rho_{2} \mu_{2}$ where $\rho_{2}$ comports with $\delta_{2}$ and $\zeta=\rho \mu$ where $\rho$ comports with $\delta$. Express the conversions of $z_{1}, z_{2}$ and $z$ from classical to rescaled as:

$$
z_{1} \sim \operatorname{SC}\left(\mu_{1}, \delta_{1}, S_{1}\right), z_{2} \sim \operatorname{SC}\left(\mu_{2}, \delta_{2}, S_{2}\right) \text { and } z \sim \operatorname{SC}\left(\mu, \delta, S_{2}\right)
$$

with respective rescaled SCD mimetics

$$
Z_{1}=R_{\mu 1 \mathrm{~S}} \Delta_{1}^{\prime} R_{\mathrm{S} \mu 1}, Z_{2}=R_{\mu 2 \mathrm{~S}} \Delta_{2}^{\prime} R_{\mathrm{S} \mu 2} \text {, and } Z=R_{\mu \mathrm{S}} \Delta^{\prime} R_{\mathrm{S} \mu} \text {. }
$$

The mimetics $Z_{1}$ and $Z_{2}$ are numerically calculated from $\left(\mu_{1}, \delta_{1}\right)$ and $\left(\mu_{2}, \delta_{2}\right)$. Their composite $Z$ is then numerically calculated as $Z=Z_{1} Z_{2}$. Let $Z_{\mathrm{E}}$ be the planar image of $Z$, and $Z_{\mathrm{m}}$ the matrix for $Z_{\mathrm{E}}$. The polar form for $Z_{\mathrm{m}}$ must be calculated as in Appendix I. The spherical mean direction $\mu$ and rescaler $\delta$ are determined from this polar form by the methods in the Appendices. See also (C) of section 4.3.

(D). SCDs are closed under rotations. Thus $z \sim \operatorname{SC}(\zeta, S)$ $\Leftrightarrow R z \sim \mathrm{SC}(R \zeta, R S)$ for any $3 \times 3$ orthogonal matrix $R$.

\section{DISCUSSION}

Selected topics outside the scope of this paper are briefly discussed in this section.

\section{(1) Uniqueness of Cauchy Directional Families}

It has been shown (see [4] for references) that the linear Cauchy distribution is the only univariate location-scale family that is closed under real Möbius transformations. [4] asserts that, consequently, any of his results in [4] are not likely to extend beyond the two-parameter Cauchy family. The CCD family may be the only family of circular probabil- 
ity distributions that is closed under changes in locationscale, although some circular distributions that are closed under these transformations bear little resemblance to the CCD. For example, the mapping

$\tan 1 / 2(\mathrm{v}-\beta)=\omega \tan 1 / 2(\mathrm{u}-\alpha),-1 \leq \omega \leq 1$

introduced by [2] as a model for circle-circle regression is indeed a one-one mapping from a u-circle onto a v-circle, but its arguments are all real and there appear to be no projections involved. Nevertheless, as shown in [2], (12) can be expressed in the general form for a circle mapping as given in (6.2).

The LCD is the only linear distribution that is closed under real Möbius transformations. Is the SCD the only spherical distribution that is closed under Möbius transformations? The answer to this is yes, by definition and by Propositions 3-6. Are there any other spherical distributions that are closed under location and scale changes? The answer to this question is unknown.

\section{(2) Higher Dimensional SCDs}

Perhaps the simplest way to generalize the SCDs to spheres $S_{\mathrm{m}}$ in m-space with (m-1)-dimensional surfaces is achieved by creating an m-vector $\zeta$ analogous to the projection point $\zeta$ in 2 - and 3 -space. Put $\zeta=r \mu$ with $|\zeta|=r \neq 1$ and with $\mu$ a unit m-vector. Define the mapping $T$ as a $\zeta$ projection, $z^{\prime}=T z$, from a unit m-sphere $S_{\mathrm{m}}$ onto itself (relabeled as $S_{\mathrm{m}}^{\prime}, S_{\mathrm{m}}^{\prime}=T_{\mathrm{m}} S_{\mathrm{m}}$ ) by means of the straight line projection making the three points $\left(z^{\prime}, z, \zeta\right)$ collinear in m-space. Then one could proceed along the lines developed in section 4.

\section{(3) Rescaling Sample Data}

Samples of directional data can be rescaled to any desired precision using rescaled or classical projections. Iterative techniques will no doubt be required in this process, since the data may generally not conform well to the symmetry, isotropy, and other features inherent in the models on which the transformations herein are based. Nevertheless, any base $\beta$ on $\mathrm{S}$ may be chosen to be rotated to the south pole, in preparation for rescaling along the $\pm \beta$ axis. Any positive value for the rescaler $\delta$ may be used. The data can be returned to their original state by undoing the operations performed in reverse order.

\section{(4) Rescaling Other Directional Distributions}

In the proof of Proposition 2 a uniformly distributed circular variable $\gamma$ was rescaled to a CCD $\gamma^{\prime}$ with the aid of (Fig. 1). The density for $\gamma^{\prime}$ was calculated to be of the form $\mathrm{f}\left(\gamma^{\prime} ; \delta, S^{\prime}\right)=\mathrm{f}(\gamma ; 1, S)\left|\mathrm{d} \gamma / \mathrm{d} \gamma^{\prime}\right|$. This procedure can be replicated using an arbitrary circular density for $\gamma$. The Jacobian $\left|\mathrm{d} \gamma / \mathrm{d} \gamma^{\prime}\right|$ is identical to that developed in the proof of Proposition 2. It depends only on the geometry of Fig. (1) and is independent of the density for $\gamma$. Perhaps some useful hybrids of the CCD and other distributions could be developed with this approach.

\section{APPENDIX I. CALCULATING POLAR FORMS}

The $k$-effect can be nullified by norming the Möbius matrix, that is, by dividing each element of $M_{\mathrm{m}}$ by the square root of the determinant. The normed matrix $N_{\mathrm{m}}=\left|M_{\mathrm{m}}\right|^{-1 / 2} M_{\mathrm{m}}$ is equivalent to the original $M_{\mathrm{m}}$ and will have determinant +1 . Since $\left|M_{\mathrm{m}}\right|$ is generally complex valued, norming forces $\operatorname{Re}\left(\left|N_{\mathrm{m}}\right|\right)=1$ and $\operatorname{Im}\left(\left|N_{\mathrm{m}}\right|\right)=0$, two restrictions which reduce the $p d f$ for the normed $N_{\mathrm{m}}$ to the required 6. Equivalent matrices for the planar Möbius function $M_{\mathrm{E}}$ are identical after they have been normed, except perhaps for algebraic sign (since if $N_{\mathrm{m}}$ is normed then $-N_{\mathrm{m}}$ is also normed, but is still equivalent to $M_{\mathrm{m}}$ ).

In calculating $w_{\mathrm{E}}$ for the Möbius function $w_{\mathrm{E}}=M_{\mathrm{E}} z_{\mathrm{E}}$ any convenient matrix equivalent by the $k$-effect to $M_{\mathrm{m}}$ can be used. But $M_{\mathrm{m}}$ must be normed before calculating polar forms, or special precautions taken, otherwise numerical errors will occur.

To illustrate the norming computations put $M_{\mathrm{m}}$ as the original Möbius matrix and $N_{\mathrm{m}}$ as the normed $M_{\mathrm{m}}$. If

$M_{\mathrm{m}}=(2-\mathrm{i}, 1+2 \mathrm{i} ; 3,2+2 \mathrm{i})$, then $\left|M_{\mathrm{m}}\right|=3-4 \mathrm{i},\left|M_{\mathrm{m}}\right|^{1 / 2}=2-\mathrm{i}$, $\left|M_{\mathrm{m}}\right|^{-1 / 2}=0.4+0.2 \mathrm{i}$, and $N_{\mathrm{m}}=\left|M_{\mathrm{m}}\right|^{-1 / 2} M_{\mathrm{m}}=(0.4+0.2 \mathrm{i}) M_{\mathrm{m}}$ $=(1, \mathrm{i} ; 1 \cdot 2+0.6 \mathrm{i}, 0.4+1.2 \mathrm{i})$, and $\left|N_{\mathrm{m}}\right|=1$.

The approach used below to get components of the polar form assumes the availability of a mathematical computer program that gets eigenvalues and eigenvectors of a $2 \times 2$ positive hermitian matrix $G_{\mathrm{m}}^{2}$. Using the normed $N_{\mathrm{m}}$ above and the formulas in section 2.6:

$G_{\mathrm{m}}^{2}=N^{*}{ }_{\mathrm{m}} N_{\mathrm{m}}=(2.8,1.2+2.2 \mathrm{i} ; 1.2-2.2 \mathrm{i}, 2.6)$, and $G_{\mathrm{m}}=$ (1.397,0.441+0.809i; 0.441-0.809i, 1.323).

$R_{\mathrm{m} \beta 0}=(0.721,-0.332-0.608 \mathrm{i} ; 0.332-0.608 \mathrm{i}, 0.721)=(\alpha, \beta ;-$ $\left.\beta^{*}, \alpha^{*}\right)$, the matrix of eigenvectors of $G_{\mathrm{m}}^{2}$ and also of $\mathrm{G}_{\mathrm{m}}$, where $R_{\mathrm{m} 0 \beta}$ and $R_{\mathrm{m} \beta 0}$ are unitary; and the base $\beta_{\mathrm{E}}=\beta / \alpha^{*}=(-$ $.332-0.608 \mathrm{i}) / 0.721=-0.46-0.844 \mathrm{i}$.

$\Delta_{\mathrm{m}}^{2}=\operatorname{Diag}(5.208,0.192)=$ the eigenvalues for $G_{\mathrm{m}}^{2} ; \Delta_{\mathrm{m}}=$ $\operatorname{Diag}(2.282,0.438)=$ the eigenvalues for $G_{\mathrm{m}}$. Note that $\left|\Delta_{\mathrm{m}}^{2}\right|$ $=\left|\Delta_{\mathrm{m}}\right|=1$, and $\delta=2.282 / 0.438=(5.208 / 0.192)^{1 / 2}=5.208$, which is coincidentally the first eigenvalue of $\Delta^{2}$.

$\mathrm{G}_{\mathrm{m}}^{-1}=R_{\mathrm{m} \beta 0} \Delta_{\mathrm{m}}^{-1} R_{\mathrm{m} 0 \beta}=(1.397,0.441+0.809 \mathrm{i} ; 0.441$ -

$0.809 \mathrm{i}, 1.323)$.

$A_{\mathrm{m}}=N_{\mathrm{m}} G_{\mathrm{m}}^{-1}=(0.515-0.441 \mathrm{i},-0.441+0.588 \mathrm{i} ; 0.441+$

$0.588 \mathrm{i}, 0.515+0.441 \mathrm{i})$, and $A_{\mathrm{m}}$ is unitary.

The planar image of the spherical mean direction $\mu$ is $\mu_{\mathrm{E}}$ $=A_{\varepsilon} \beta_{\mathrm{E}}=-1.327+0.111 \mathrm{i}($ not $\tau$-corrected $)$. The spherical image $\mu=\tau \mathrm{A} \beta=(0.957,-0.08,-0.279)^{\mathrm{T}}(\tau$-corrected $)$.

\section{APPENDIX II. GAUSS' ROTATION THEOREM}

To express the relationships between the parameters of $A_{\mathrm{m}}$ and $A$, where $A \leftrightarrow A_{\mathrm{E}}$, recall that every unitary matrix $A_{\mathrm{m}}$ has two complex parameters $(\alpha, \beta)$, and we write: $A_{\mathrm{m}}=(\alpha, \beta)$. Also recall that every $3 \times 3$ proper rotation matrix $A$ is uniquely determined by three angles, $(\eta, \theta, \varphi)$, and we write: $A=(\eta, \theta, \varphi)$, where

$\eta=$ amount of rotation, $0<\eta \leq \pi$ (if $\eta=0$ then $A=I_{3}$, the identity matrix),

$p_{\mathrm{S}}=(\theta, \varphi)=$ spherical coordinates for the positive axis of rotation, $p$, where $p^{\mathrm{T}} p=1$ and 
$p=(x, y, t)^{\mathrm{T}}=(\sin \theta \cos \varphi, \sin \theta \sin \varphi, \cos \theta)^{\mathrm{T}}$.

The $3 \times 3$ rotation matrices of the extended pseudo-polar form of a spherical Möbius function $M$ must all necessarily be proper. The improper case was used to account for negative correlation in spherical regression by [2], and is included here for completeness.

If $A$ is improper then $A J$ is proper and can be described by $A J=(\eta, \theta, \varphi)$, where $J=\operatorname{Diag}(1,-1,1)$. The $3 \times 3$ improper $A$ $=\left(\mathrm{a}_{\mathrm{ij}}\right)$ can be recovered from $A J$ by the identity $A \equiv(A J) J$. The stage is now set for providing the details of Gauss' Rotation image theorem and its stunning results:

Gauss' rotation image theorem (A): Assume that $A$ is proper and $A=(\eta, \theta, \varphi)$, and $\mathrm{A}_{\mathrm{m}}$ is unitary and $A_{\mathrm{m}}=(\alpha, \beta)$. Get $(\eta, \theta, \varphi)$ from $(\alpha, \beta)$ by:

$\cos \eta / 2=x_{\alpha}, \alpha=x_{\alpha}+\mathrm{i} y_{\alpha}, 0<\eta / 2 \leq \pi / 2 ;$

$\cos \theta=y_{\alpha} / \sin \eta / 2,0 \leq \theta \leq \pi$;

$\cos \varphi=y_{\beta} /\left(x_{\beta}^{2}+y_{\beta}^{2}\right)^{1 / 2}, \beta=x_{\beta}+\mathrm{i} y_{\beta},-\pi<\varphi \leq \pi ;$

$\sin \varphi=x_{\beta} /\left(x_{\beta}^{2}+y_{\beta}^{2}\right)^{1 / 2}$.

For the $A_{\mathrm{m}}=(\alpha, \beta)=(0.515-0.441 \mathrm{i},-0.441+0.588 \mathrm{i})$ of Appendix I:

$A_{\mathrm{m}}=\left(\alpha, \beta ;-\beta^{*}, \alpha^{*}\right)=(0.515-0.441 \mathrm{i},-0.441+0.588 \mathrm{i} ;$

$0.441+0.588 \mathrm{i}, 0.515+0.441 \mathrm{i})$.

Using (A) above we get the three angles of $A$ in radians:

$A=(\eta, \theta, \varphi)=(2.06,2.111,-0.644)$.

Gauss' rotation image theorem (B): Get unitary complex parameters $(\alpha, \beta)$ from proper rotation angles $(\eta, \theta, \varphi)$ by

$x_{\alpha}=\cos \eta / 2, y_{\alpha}=\cos \theta \sin \eta / 2 ; x_{\beta}=\sin \theta \sin \varphi \sin \eta / 2$, $y_{\beta}=\sin \theta \cos \varphi \sin \eta / 2$.

Applying (B) to $(\eta, \theta, \varphi)$ of $A$ we get the $(\alpha, \beta)$ of $A_{\mathrm{m}}$ that we obtained in Appendix I.

Gauss' rotation image theorem (C): If $A$ is an improper spherical rotation use $A J$ and $A_{\mathrm{E}} z_{\mathrm{E}}$ * where

$A_{\mathrm{E}} z_{\mathrm{E}} * \leftrightarrow A J z$ whenever $z_{\mathrm{E}} \leftrightarrow z$ and $A_{\mathrm{E}} \leftrightarrow A$

Equations for (A) and (B) above are adapted from [1, p. 286-290], who gives an insightful partial proof of Gauss' rotation theorem, amply illustrated with figures. [12] gives a compact but more complete proof.

Conversions back and forth between unitary matrices and various forms of rotation matrices are essential to analyses herein but tedious to assemble from reference books, so algorithmic forms for calculating the 9 elements of a proper rotation matrix $\mathrm{A}=\left(\mathrm{a}_{\mathrm{ij}}\right)$ from the three angles for $\mathrm{A}=$ $[\eta, \theta, \varphi]$, and conversely, are presented below.

(D) Get the 3 rotation angles $(\eta, \theta, \varphi)$ from the 9 matrix elements of a proper rotation $A=\left(a_{i j}\right)$ by

$\eta=\operatorname{acos}\{1 / 2(\operatorname{tr} A-1)\}, \quad-\pi<\eta \leq \pi$,

$\theta=\operatorname{acos}\left\{\left(a_{21}-a_{12}\right) /(2 \sin \eta)\right\}, 0 \leq \theta \leq \pi$, $\varphi=\operatorname{acos}\left[\left(a_{32}-a_{23}\right) /\left\{\left(a_{32}-a_{23}\right)^{2}+\left(a_{13}-a_{31}\right)^{2}\right\}^{1 / 2}\right]$

where $0 \leq \varphi \leq \pi$ if $a_{13} \geq a_{31},-\pi<\varphi<0$ if $a_{13}<a_{31}$.

Equations for (D) are adapted from [13.1]. If $\mathrm{a}_{33} \approx 1$ then the positive rotation axis for $A$ is $n, \theta$ is zero, $\varphi$ does not exist, and it is not necessary to use (D).

(E) Get the 9 matrix elements $\mathrm{a}_{\mathrm{ij}}$ from the three proper rotation angles $(\eta, \theta, \varphi)$ by

$A=\cos \eta\left(\mathrm{I}_{3}-p p^{\mathrm{T}}\right)+(1-\cos \eta) p p^{\mathrm{T}}+\sin \eta K$,

where $K$ is a $3 \times 3$ skew-symmetric matrix with $1^{\text {st }}$ row $(0,-t, y), 2^{\text {nd }}$ row $(t, 0,-x), 3^{\text {rd }}$ row $(-y, x, 0)$, and $p=(x, y, t)^{\mathrm{T}}=$ rectangular coordinates for the positive axis of rotation, with spherical coordinates $p_{\mathrm{S}}=\left(\theta_{\mathrm{p}}, \varphi_{\mathrm{p}}\right)$. The equation for $(\mathrm{E})$ is adapted from [13.2].

Applying (E) to the $(\eta, \theta, \varphi)$ results of $(A)$ for $A_{m}$ we get the three rows of the $3 \times 3$ proper rotation matrix $A$ : the $1^{\text {st }}$ row is $(-0.33,-0.94,-0.091), 2^{\text {nd }}$ row $(0.473,-0.081,-0.878)$ and $3^{\text {rd }}$ row $(0.817,-0.333,0.471)$.

The rotor $R_{\mathrm{E} \beta 0}$ of section 2.2 has spherical image $R_{\beta \mathrm{S}}$ defined by the condition $\beta=R_{\beta S}$, the proper rotation which sends $s$ to the spherical base point $\beta$ along the meridian of $\beta$, and simultaneously sends $-s$ to $-\beta$. Equals have equal images, so the planar image of $\left(\beta=\mathrm{R}_{\beta \mathrm{S}} \mathrm{S}\right)$ is $\left(\beta_{\mathrm{E}}=R_{\mathrm{E} \beta 0} 0\right)$. This information was used to get $\beta_{\mathrm{E}}$ in the numerical example of Appendix I. Put $\mathrm{R}_{\mathrm{m} \beta 0}=\left(\alpha, \beta ;-\beta^{*}, \alpha^{*}\right)$ as the matrix for $\mathrm{R}_{\mathrm{E} \beta 0}$. Then $R_{\mathrm{E} \beta 0} 0=\beta_{\mathrm{E}}$ implies $\beta_{\mathrm{E}}=\beta / \alpha^{*}$. For the numerical example in Appendix I we get

$R_{\mathrm{m} \beta 0}=(0.721,-0.332-0.608 \mathrm{i} ; 0.332-0.608 \mathrm{i}, 0.721)$, so

$\beta_{\mathrm{E}}=\beta / \alpha^{*}=(-0.332-0.608 \mathrm{i}) / 0.721=-0.46-0.844 \mathrm{i}$

\section{REFERENCES}

[1] T. Needham, Visual Complex Analysis. New York: Oxford University Press, 1998.

[2] T. D. Downs, and K. V. Mardia, "Circular regression", Biometrika, vol. 89, pp. 683-697, August 2002.

[3] T. D. Downs, "Spherical regression", Biometrika, vol. 90, pp. 655668, September 2003.

[4] P. McCullagh, "Möbius transformation and Cauchy parameter estimation", Annals Statist., vol. 24, No. 2, pp. 787- 808, 1996. [Online] Available: http:// projecteuclid.org/aos [Accessed Dec. 4, 2008].

[5] K. Knopp, Elements of the Theory of Functions. F. Bagemihl, Translator. New York: Dover, 1952, pp. 42-65.

[6] H. Schwerdtfeger, Geometry of Complex Numbers. New York: Dover, 1979.

[7] C. F. Gauss, Unpublished Works, c. 1819.

[8] K. V. Mardia, and P. E. Jupp, Directional Statistics. Chichester: Wiley, 2000

[9] K. Shogo, K. Shimizu, and G. S. Shieh, "A circular- circular regression model”. Stat. Sinica, vol. 18, pp. 633-645, April 2008.

[10] L. Hahn, Complex Numbers and Geometry. Mathematical Association of America: USA, 2002, pp. 135-137.

[11] T.D. Downs, "Some relationships among the von Mises distributions of different dimensions", Biometrika, vol. 53, pp. 269-272, June 1966. 
[12] J. Stilwell, Geometry of Surfaces. New York: Springer, 1992, pp. 57-59.
[13]

G. A. Korn, and T. M. Korn, Mathematical Handbook for Scientists and Engineers. New York: Dover, 2000, pp. 472-473.

Received: June 01, 2009

(C) T.D. Downs; Licensee Bentham Open.

This is an open access article licensed under the terms of the Creative Commons Attribution Non-Commercial License (http://creativecommons.org/licenses/ by-nc/3.0/) which permits unrestricted, non-commercial use, distribution and reproduction in any medium, provided the work is properly cited. 